\title{
Characterisation of homogeneous fractional Sobolev spaces
}

\author{
Lorenzo Brasco $^{1}$ • David Gómez-Castro ${ }^{2,3}$. Juan Luis Vázquez ${ }^{4}$
}

Received: 21 July 2020 / Accepted: 5 February 2021 / Published online: 1 March 2021

(c) The Author(s) 2021

\begin{abstract}
Our aim is to characterize the homogeneous fractional Sobolev-Slobodeckiu spaces $\mathcal{D}^{s, p}\left(\mathbb{R}^{n}\right)$ and their embeddings, for $s \in(0,1]$ and $p \geq 1$. They are defined as the completion of the set of smooth and compactly supported test functions with respect to the Gagliardo-Slobodeckir seminorms. For $s p<n$ or $s=p=n=1$ we show that $\mathcal{D}^{s, p}\left(\mathbb{R}^{n}\right)$ is isomorphic to a suitable function space, whereas for $s p \geq n$ it is isomorphic to a space of equivalence classes of functions, differing by an additive constant. As one of our main tools, we present a Morrey-Campanato inequality where the Gagliardo-Slobodeckiǔ seminorm controls from above a suitable Campanato seminorm.
\end{abstract}

\section{Mathematics Subject Classification 46E35}

\section{Contents}

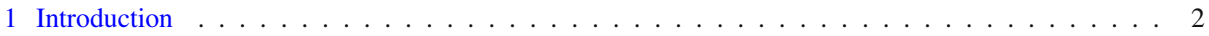

1.1 Fractional Sobolev spaces . . . . . . . . . . . . . . . . . . . . 2

1.2 Motivation for the homogeneous Sobolev spaces . . . . . . . . . . . . . . . . . 3

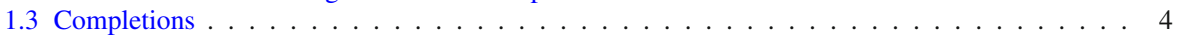

1.4 Main results: the three ranges . . . . . . . . . . . . . . . . . . . . . . . . 5

Communicated by A. Malchiodi.

$\triangle$ Lorenzo Brasco

lorenzo.brasco@unife.it

David Gómez-Castro

gomezcastro@maths.ox.ac.uk

Juan Luis Vázquez

juanluis.vazquez@uam.es

1 Dipartimento di Matematica e Informatica, Università degli Studi di Ferrara, Via Machiavelli 30, 44121 Ferrara, Italy

2 Mathematical Institute, University of Oxford, Radcliffe Observatory Quarter, Woodstock Road, Oxford OX2 6GG, UK

3 Instituto de Matemática Interdisciplinar, Universidad Complutense de Madrid, Plaza de Ciencias 3 , 28040 Madrid, Spain

4 Departamento de Matemáticas, Universidad Autónoma de Madrid, Ciudad Universitaria de Cantoblanco, 28049 Madrid, Spain 


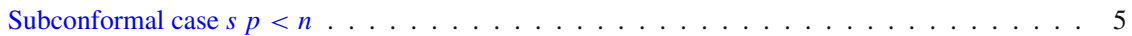

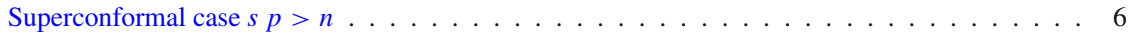

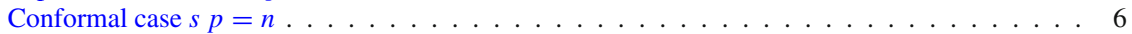

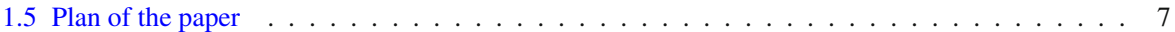

2 Preliminaries .............................. 8

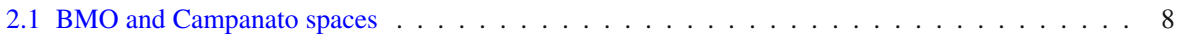

2.2 Weighted integrability for some Campanato spaces . . . . . . . . . . . . . . . . . 9

2.3 A Morrey-Campanato-type inequality and applications . . . . . . . . . . . . . . . . . 11

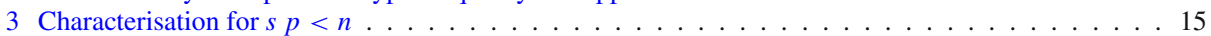

4 Characterisation for $s p>n \ldots \ldots \ldots \ldots \ldots \ldots$

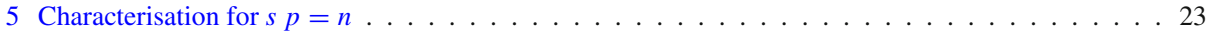

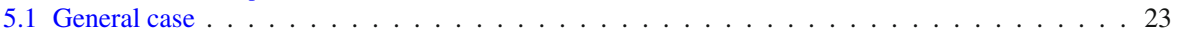

5.2 The exceptional limit case $s=n=1 \ldots \ldots \ldots \ldots \ldots \ldots \ldots \ldots$. . . . . . . . . . . . . . . . . . . . . . . .

6 Comments and open questions . . . . . . . . . . . . . . . . . . . . . . . . . . . 28

Appendix A. Approximation by convolution . . . . . . . . . . . . . . . . . . . . . . . . . . . 29

Appendix B. Truncation lemmas . . . . . . . . . . . . . . . . . . . . . . . 30

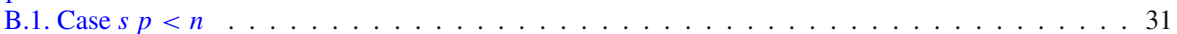

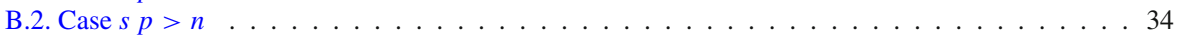

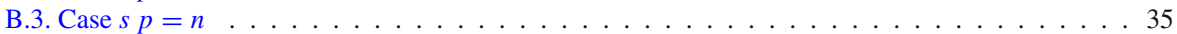

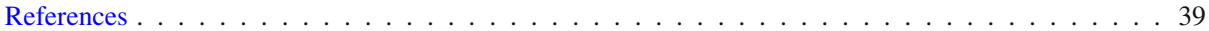

\section{Introduction}

\subsection{Fractional Sobolev spaces}

The aim of this paper is to shed light on an important topic in the theory of fractional Sobolev spaces. This family of spaces is conveniently presented e.g. in $[1,17,19,29]$. It is common to define the fractional Sobolev spaces $W^{s, p}\left(\mathbb{R}^{n}\right)$ in the Sobolev-Slobodeckil form. Thus, for $s \in(0,1)$ and $1 \leq p<+\infty$ we define the normalized Gagliardo-Slobodeckiŭ seminorm by

$$
[u]_{W^{s, p}\left(\mathbb{R}^{n}\right)}=\left(s(1-s) \iint_{\mathbb{R}^{n} \times \mathbb{R}^{n}} \frac{|u(x)-u(y)|^{p}}{|x-y|^{n+s p}} d x d y\right)^{\frac{1}{p}} .
$$

Then,

$$
W^{s, p}\left(\mathbb{R}^{n}\right):=\left\{u \in L^{p}\left(\mathbb{R}^{n}\right):[u]_{W^{s, p}\left(\mathbb{R}^{n}\right)}<+\infty\right\},
$$

is a Banach space endowed with the non-homogeneous norm

$$
\|u\|_{W^{s, p}\left(\mathbb{R}^{n}\right)}=\|u\|_{L^{p}\left(\mathbb{R}^{n}\right)}+[u]_{W^{s, p}\left(\mathbb{R}^{n}\right)} .
$$

A word about the convenience of the extra factor $s(1-s)$ is in order. Indeed, $[\cdot]_{W^{s, p}}\left(\mathbb{R}^{n}\right)$ can be thought as a real interpolation quantity, with parameter $s$, between the two quantities

$$
\int_{\mathbb{R}^{n}}|u|^{p} d x \text { and } \int_{\mathbb{R}^{n}}|\nabla u|^{p} d x
$$

see for example [6] or [9]. It is then natural to expect the following asymptotic behaviour

$$
\iint_{\mathbb{R}^{n} \times \mathbb{R}^{n}} \frac{|u(x)-u(y)|^{p}}{|x-y|^{n+s p}} d x d y \sim \frac{C}{s} \int_{\mathbb{R}^{n}}|u|^{p} d x, \quad \text { for } s \searrow 0,
$$

and

$$
\iint_{\mathbb{R}^{n} \times \mathbb{R}^{n}} \frac{|u(x)-u(y)|^{p}}{|x-y|^{n+s p}} d x d y \sim \frac{C}{1-s} \int_{\mathbb{R}^{n}}|\nabla u|^{p} d x, \text { for } s \nearrow 1,
$$


see [20] for the first result and [4] for the second one. For this reason, the factor $s(1-s)$ is incorporated in the definition of the seminorm and the limit cases $s=0$ and $s=1$ are defined accordingly by

$$
[u]_{W^{0, p}\left(\mathbb{R}^{n}\right)}=\|u\|_{L^{p}\left(\mathbb{R}^{n}\right)} \quad \text { and } \quad[u]_{W^{1, p}\left(\mathbb{R}^{n}\right)}=\|\nabla u\|_{L^{p}\left(\mathbb{R}^{n}\right)} .
$$

There are well-known embeddings of these spaces $W^{s, p}\left(\mathbb{R}^{n}\right)$ into $L^{q}\left(\mathbb{R}^{n}\right)$ for suitable $q \geq 1$ for which we refer to the classical monographs like [1,17].

The particular case $s=(p-1) / p$ has a peculiar theoretical importance, since in this case

$$
W^{\frac{p-1}{p}, p}\left(\mathbb{R}^{n}\right)
$$

can be identified with the trace space of functions in $W^{1, p}\left(\mathbb{H}_{+}^{n+1}\right)$, where $\mathbb{H}_{+}^{n+1}=\mathbb{R}^{n} \times$ $[0,+\infty)$. More generally, it can be proved that $W^{s, p}\left(\mathbb{R}^{n}\right)$ coincides with the trace space of the weighted Sobolev space $\mathcal{W}_{s}^{1, p}\left(\mathbb{H}_{+}^{n+1}\right)$, defined as

$\mathcal{W}_{s}^{1, p}\left(\mathbb{H}_{+}^{n+1}\right)=\left\{u \in L_{\mathrm{loc}}^{1}\left(\mathbb{H}_{+}^{n+1}\right): u y^{\frac{(p-1)-s p}{p}} \in L^{p}\left(\mathbb{H}_{+}^{n+1}\right),|\nabla u| y^{\frac{(p-1)-s p}{p}} \in L^{p}\left(\mathbb{H}_{+}^{n+1}\right)\right\}$,

where we have used the notation $(x, y) \in \mathbb{H}_{+}^{n+1}$, with $x \in \mathbb{R}^{n}$ and $y \in[0,+\infty)$. See [18, Section 5] for more details. The reader may also consult the recent paper [22], containing some generalizations.

\subsection{Motivation for the homogeneous Sobolev spaces}

Before we present the main results of this paper, we discuss some motivations for the study of a particular class of fractional Sobolev spaces. Recently, there has been a surge of interest towards the study of nonlocal elliptic operators, that arise as first variations of GagliardoSlobodeckii seminorms. The leading example is given by the fractional Laplacian of order $s$ of a function $u$, indicated by the symbol $(-\Delta)^{s} u$, which in weak form reads as

$$
\left\langle(-\Delta)^{s} u, \varphi\right\rangle=\iint_{\mathbb{R}^{n} \times \mathbb{R}^{n}} \frac{(u(x)-u(y))(\varphi(x)-\varphi(y))}{|x-y|^{n+2 s}} d x d y,
$$

for all $\varphi \in C_{c}^{\infty}\left(\mathbb{R}^{n}\right)$, up to a possible normalization factor. Observe that this is nothing but the first variation of the functional

$$
u \mapsto \frac{1}{2}[u]_{W^{s, 2}\left(\mathbb{R}^{n}\right)}^{2},
$$

up to the factor $s(1-s)$. More generally, one could take a general exponent $1<p<+\infty$ and obtain accordingly the fractional p-Laplacian of order $s$ of a function $u$, which we denote $\left(-\Delta_{p}\right)^{s} u$, and is defined in weak form by

$$
\left\langle\left(-\Delta_{p}\right)^{s} u, \varphi\right\rangle=\iint_{\mathbb{R}^{n} \times \mathbb{R}^{n}} \frac{|u(x)-u(y)|^{p-2}(u(x)-u(y))(\varphi(x)-\varphi(y))}{|x-y|^{n+s p}} d x d y,
$$

for all $\varphi \in C_{c}^{\infty}\left(\mathbb{R}^{n}\right)$, up to a multiplicative factor. This is a nonlocal and nonlinear operator which has been extensively studied in recent years, see [31,32] and references therein. In order to motivate the studies performed in this paper, let us consider the quasilinear nonlocal elliptic problem

$$
\left(-\Delta_{p}\right)^{s} u=f, \quad \text { in } \mathbb{R}^{n}
$$


under suitable assumptions on the source term $f$. In order to prove existence of a weak solution, it would be natural to use the Direct Method in the Calculus of Variations. This would lead to the problem of minimizing the energy functional

$$
u \mapsto \frac{1}{p}[u]_{W^{s, p}\left(\mathbb{R}^{n}\right)}^{p}-\int_{\mathbb{R}^{n}} f u d x,
$$

which is naturally associated to our equation. However, it is not clear the functional space where this minimization problem should be posed. For example, one could try to pose the problem in the previously introduced space $W^{s, p}\left(\mathbb{R}^{n}\right)$, but it is easily seen that this does not fit at all. Indeed, the functional (1.2) is not weakly coercive on this space, unless we are in the trivial situation $f \equiv 0$. Of course, the problem is that the functional (1.2) can not permit to infer any control on the $L^{p}$ norm of minimizing sequences. This in turn is related to the fact that the Poincaré inequality

$$
c \int_{\mathbb{R}^{n}}|u|^{p} d x \leq[u]_{W^{s, p}\left(\mathbb{R}^{n}\right)}^{p}
$$

fails to be true on the whole $\mathbb{R}^{n}$ for any $c>0$. This can be easily seen by using the invariance of $\mathbb{R}^{n}$ with respect to scalings $x \mapsto \lambda x$ and a simple dimensional analysis of the two norms.

It turns out that the natural spaces to work with are the homogeneous Sobolev spaces $\mathcal{D}^{s, p}\left(\mathbb{R}^{n}\right)$. They are defined by

$$
\mathcal{D}^{s, p}\left(\mathbb{R}^{n}\right):=\text { "completion of } C_{c}^{\infty}\left(\mathbb{R}^{n}\right) \text { with respect to }[\cdot]_{W^{s, p}}\left(\mathbb{R}^{n}\right) \text { ". }
$$

We recall that $W^{s, p}\left(\mathbb{R}^{n}\right)$ and $\mathcal{D}^{s, p}\left(\mathbb{R}^{n}\right)$ are particular instances of the huge family of Besov spaces. In this respect, we stress that a mention of homogeneous Besov spaces can be found for example in [1, Remark 7.68], [3, Chapter 6, Section 3], [19, Chapter 10, Section 1] and [30, Chapter 3, Section 4], among others.

The notation $\mathcal{D}^{s, p}\left(\mathbb{R}^{n}\right)$ adopted here is reminiscent of the historical one, introduced by Deny and Lions in their paper [10]. This reference has been among the first papers to study homogeneous spaces, obtained by completion of $C_{c}^{\infty}\left(\mathbb{R}^{n}\right)$.

Another frequently encountered notation for homogeneous Sobolev spaces is $\dot{W}^{s, p}\left(\mathbb{R}^{n}\right)$, see for example Petree's paper [26]. However, usually this notation is used for spaces of functions identified modulo constants for $s \in(0,1]$. We will adopt the same convention in this paper.

It is our aim to examining the space $\mathcal{D}^{s, p}\left(\mathbb{R}^{n}\right)$ more closely and connect it with spaces of the type $\dot{W}^{s, p}\left(\mathbb{R}^{n}\right)$, as we will explain in a moment.

\subsection{Completions}

Before presenting the main results of the paper, let us briefly recall some basic facts about the completion process. We start from the seminorm $[\cdot]_{W^{s, p}\left(\mathbb{R}^{n}\right)}$ for $0<s \leq 1$ and $1<p<\infty$. It is not difficult to see that this turns out to be a norm on the space $C_{c}^{\infty}\left(\mathbb{R}^{n}\right)$.

However, the normed space

$$
\left(C_{c}^{\infty}\left(\mathbb{R}^{n}\right),[\cdot]_{W^{s, p}\left(\mathbb{R}^{n}\right)}\right),
$$

is not complete. By definition, its completion is the quotient space of the set of sequences $\left(u_{m}\right)_{m \in \mathbb{N}} \subset C_{c}^{\infty}\left(\mathbb{R}^{n}\right)$ which are Cauchy for the norm $[\cdot]_{W^{s, p}\left(\mathbb{R}^{n}\right)}$, under the expected equivalence relation

$$
\left(u_{m}\right)_{m \in \mathbb{N}} \sim_{s, p}\left(v_{m}\right)_{m \in \mathbb{N}} \text { if } \quad \lim _{m \rightarrow \infty}\left[u_{m}-v_{m}\right]_{W^{s, p}\left(\mathbb{R}^{n}\right)}=0 .
$$


For each equivalence class $U=\left\{\left(u_{m}\right)_{m \in \mathbb{N}}\right\}_{s, p} \in \mathcal{D}^{s, p}\left(\mathbb{R}^{n}\right)$, we define its norm in terms of a representative as

$$
\|U\|_{\mathcal{D}^{s, p}\left(\mathbb{R}^{n}\right)}:=\lim _{m \rightarrow \infty}\left[u_{m}\right]_{W^{s, p}\left(\mathbb{R}^{n}\right)} .
$$

It is easily seen that such a definition is independent of the chosen representative. With this construction,

$$
\left(\mathcal{D}^{s, p}\left(\mathbb{R}^{n}\right),\|\cdot\|_{\mathcal{D}^{s, p}\left(\mathbb{R}^{n}\right)}\right)
$$

is a Banach space. Note that all functions $u \in C_{c}^{\infty}\left(\mathbb{R}^{n}\right)$ can be naturally embedded into $\mathcal{D}^{s, p}\left(\mathbb{R}^{n}\right)$ by the constant sequence $u_{m}=u$. By definition, this representation of $C_{c}^{\infty}\left(\mathbb{R}^{n}\right)$ is dense in $\mathcal{D}^{s, p}\left(\mathbb{R}^{n}\right)$.

In the case $s=1$, it is well-known that $\mathcal{D}^{1, p}\left(\mathbb{R}^{n}\right)$ is a subspace of the space of distributions $\mathcal{D}^{\prime}\left(\mathbb{R}^{n}\right)$ if and only if $1 \leq p<n$. In this case, this can be identified with the functional space

$$
\left\{u \in L^{p^{*}}\left(\mathbb{R}^{n}\right): \nabla u \in L^{p}\left(\mathbb{R}^{n} ; \mathbb{R}^{n}\right)\right\}, \quad \text { where } p^{*}=\frac{n p}{n-p},
$$

thanks to the celebrated Sobolev inequality. The case $p=2$ is contained in Deny and Lions, [10, Théorème 4.4 and Remark 4.1]. The general case can be found for example in [19, Chapter 15]. On the contrary, the case $p \geq n$ is much more delicate, since in this case $\mathcal{D}^{1, p}\left(\mathbb{R}^{n}\right)$ is not even a subspace of $\mathcal{D}^{\prime}\left(\mathbb{R}^{n}\right)$. A concrete characterization of $\mathcal{D}^{1, p}\left(\mathbb{R}^{n}\right)$ as a space of equivalence classes of functions modulo constants seems to belong to the folklore on the subject, though we have not been able to find a proper reference in the literature. Our presentation will cover this case, as well.

\subsection{Main results: the three ranges}

The main question we address in these notes is the characterization of $\mathcal{D}^{s, p}\left(\mathbb{R}^{n}\right)$ and the study of some of its embeddings into suitable sets of functions. This will be possible for some exponents, while for other exponents the embedding occurs into a space of equivalence classes of functions modulo constants, as we are now going to explain.

More precisely, in order to answer to these questions, we will need to distinguish three cases, according to the different behaviours of

$$
u \mapsto[u]_{W^{s, p}\left(\mathbb{R}^{n}\right)},
$$

with respect to scalings of the form $x \mapsto \lambda x$, with $\lambda>0$. By a simple change in variable, we have that

$$
\left[u_{\lambda}\right]_{W^{s, p}\left(\mathbb{R}^{n}\right)}=\lambda^{s-\frac{n}{p}}[u]_{W^{s, p}\left(\mathbb{R}^{n}\right)}, \quad \text { for every } \lambda>0, \text { where } u_{\lambda}(x)=u(\lambda x),
$$

This shows that the relation between $s p$ and $n$ provides significantly different results. Consequently, there are three different situations:

\section{Subconformal case $s p<n$}

The natural inclusion of $\mathcal{D}^{s, p}\left(\mathbb{R}^{n}\right)$ into a Lebesgue space of functions succeeds. More precisely, the completion can be identified with a functional space, i.e., we have

$\mathcal{D}^{s, p}\left(\mathbb{R}^{n}\right) \simeq \dot{W}^{s, p}\left(\mathbb{R}^{n}\right):=\left\{u \in L^{p_{s}^{\star}}\left(\mathbb{R}^{n}\right):[u]_{W^{s, p}\left(\mathbb{R}^{n}\right)}<+\infty\right\}, \quad$ where $p_{s}^{\star}=\frac{n p}{n-s p}$, 
see Theorem 3.1 below. The main tool here is the fractional Sobolev inequality. This mimics in some sense what happens for $W^{s, p}\left(\mathbb{R}^{n}\right)$, that can be characterized by (1.1) as the completion of $C_{c}^{\infty}\left(\mathbb{R}^{n}\right)$ with respect to the $W^{s, p}\left(\mathbb{R}^{n}\right)$ norm.

\section{Superconformal case $s p>n$}

Here, the Sobolev inequality is not available and we have to replace it by Morrey's inequality, see Eq. (2.11) below. However, unlike the case $s p<n$, the elements in $\mathcal{D}^{s, p}\left(\mathbb{R}^{n}\right)$ can not be uniquely represented by functions. Indeed, when $s p>n$, there exist sequences $\left(\varphi_{m}\right)_{m \in \mathbb{N}}$ such that

$$
\left[\varphi_{m}\right]_{W^{s, p}\left(\mathbb{R}^{n}\right)} \rightarrow 0 \text { and } \quad \varphi_{m} \rightarrow 1 \text { uniformly over compact sets, }
$$

as $m$ goes to $\infty$. These sequences are known as null-sequences. Hence, any sequence $\left(u_{m}\right)_{m \in \mathbb{N}} \subset C_{c}^{\infty}\left(\mathbb{R}^{n}\right)$ which is Cauchy in the norm $[\cdot]_{W^{s, p}\left(\mathbb{R}^{n}\right)}$ is equivalent to the sequence

$$
v_{m}=u_{m}+C \varphi_{m},
$$

for any constant $C \in \mathbb{R}$. Observe that this implies in particular that now all constant functions are equivalent to the null one in $\mathcal{D}^{s, p}\left(\mathbb{R}^{n}\right)$.

Furthermore, one can show that functions that are approximated by equivalent Cauchy sequences actually coincide up to a constant. This allows to show that $\mathcal{D}^{s, p}\left(\mathbb{R}^{n}\right)$ can be identified with a space of equivalence classes of Hölder continuous functions differing by an additive constant, i.e. we have

$$
\mathcal{D}^{s, p}\left(\mathbb{R}^{n}\right) \simeq \dot{W^{s, p}}\left(\mathbb{R}^{n}\right):=\frac{\left\{u \in C^{0, s-\frac{n}{p}}\left(\mathbb{R}^{n}\right):[u]_{W^{s, p}\left(\mathbb{R}^{n}\right)}<+\infty\right\}}{\sim_{C}},
$$

where for $0<\alpha \leq 1$

$$
C^{0, \alpha}\left(\mathbb{R}^{n}\right)=\left\{u: \mathbb{R}^{n} \rightarrow \mathbb{R}: \sup _{x \neq y} \frac{|u(x)-u(y)|}{|x-y|^{\alpha}}<+\infty\right\},
$$

and $\sim_{C}$ is the equivalence relation

$$
u \sim_{C} v \Longleftrightarrow u-v \text { is constant. }
$$

We refer to Theorem 4.4 below, for complete details.

\section{Conformal case $s p=n$}

This is the most delicate case. Whenever $s<n$ (i. e., unless $s=n=1$ ), it is still possible to prove existence of a sequence $\left(\varphi_{m}\right)_{m \in \mathbb{N}}$ such that properties (1.4) hold. However, the construction of such a sequence is now more involved. Since the seminorm is scale invariant in this case, such a construction can not be just based on scalings. As in the local case $s=1$, one has to consider a suitable sequence of truncated and rescaled logarithms (see Lemma 5.1 below). This is reminiscent of the optimal sequence for the Moser-Trudinger inequality, see for example [25, Section 5] for the fractional case.

This permits to show that also in the case $s p=n$ (provided $s<n$ ), we can approximate the constant functions by functions in the null class. Hence, this case behaves like $s p>n$ 
and $\mathcal{D}^{s, n / s}\left(\mathbb{R}^{n}\right)$ can be identified with a space of equivalence classes of $B M O$ functions differing by an additive constant, i.e. we have

$$
\mathcal{D}^{s, \frac{n}{s}}\left(\mathbb{R}^{n}\right) \simeq \dot{W}^{s, \frac{n}{s}}\left(\mathbb{R}^{n}\right):=\frac{\left\{u \in B M O\left(\mathbb{R}^{n}\right):[u]_{W^{s, \frac{n}{s}}\left(\mathbb{R}^{n}\right)}<+\infty\right\}}{\sim_{C}},
$$

see Theorem 5.3 below.

Still, there will be room for a small surprise. Indeed, we will show that the limiting case $s=p=n=1$, which still falls in the conformal regime, behaves like $s p<n$. In other words, the homogeneous Sobolev space $\mathcal{D}^{1,1}(\mathbb{R})$ is actually a function space and we have

$$
\mathcal{D}^{1,1}(\mathbb{R}) \simeq \dot{W}^{1,1}\left(\mathbb{R}^{n}\right):=\left\{u \in C_{0}(\mathbb{R}): \int_{\mathbb{R}}\left|u^{\prime}\right| d x<+\infty\right\},
$$

where $C_{0}(\mathbb{R})$ is the space of continuous functions vanishing at infinity, see Theorem 5.5 below. In this way we complete the characterization of the spaces.

Remark 1.1 After completing this work, we became aware of the interesting recent paper [23], dealing with the same issue here addressed, but for a different scale of fractional Sobolev spaces. Namely, the authors of [23] deal with the so-called Bessel potential spaces (sometimes also called Liouville spaces), which are defined in terms of the Fourier transform. In [23, Theorem 2], they give a concrete realization of the homogeneous version of these spaces.

Our results only partially superpose with those of [23] and, in any case, the proofs are different.

Indeed, the results of [23] are based on Harmonic Analysis techniques and contain ours only for the case $p=2$ and $0<s<1$, and for $1<p<\infty$ and $s=1$.

\subsection{Plan of the paper}

We start with Sect. 2, where some basic facts about $B M O$ and Campanato spaces are recalled. These tools are particularly useful to handle the cases $s p \geq n$. In this part, an important result is Theorem 2.4, which relates the Gagliardo-Slobodeckii seminorm and a Campanato seminorm. This yields as corollaries several important inequalities: a fractional PoincaréWirtinger inequality (see Corollary 2.5) and the fractional Morrey inequality for $s p>n$ (see Corollary 2.7).

We devote Sects. 3, 4 and 5 to prove the characterisation of $\mathcal{D}^{s, p}$ in the cases $s p<n$, $s p>n$, and $s p=n$, respectively. We introduce in each case suitable structural lemmas.

We conclude the paper with two appendices on approximation lemmas, which will allow to show that the elements in $\dot{W}^{s, p}\left(\mathbb{R}^{n}\right)$ can be approximated by functions in $C_{c}^{\infty}\left(\mathbb{R}^{n}\right)$. The aim is to approximate a function $u$ in the Gagliardo-Slobodeckir seminorm by sequences of the type $\left(u * \rho_{m}\right) \eta_{m}$, where $\rho_{m}$ are standard mollifiers and $\eta_{m}$ are cut-off functions. In Appendix A we study the convolution, while in Appendix B we prove several truncation lemmas, which allow to estimate the effect of multiplying by cut-off functions. 


\section{Preliminaries}

\subsection{BMO and Campanato spaces}

At first, we need to recall definitions and some basic facts about bounded mean oscillation functions and Campanato spaces. As already announced, this will be particularly useful to deal with the cases $s p>n$ and $s p=n$. We will indicate by $B_{r}\left(x_{0}\right)$ the $n$-dimensional open ball with center $x_{0} \in \mathbb{R}^{n}$ and radius $r>0$. The symbol $\omega_{n}$ will stand for the measure of $B_{1}(0)$.

A function of bounded mean oscillation is a locally integrable function $u$ such that the supremum of its mean oscillations is finite. More precisely, for every $u \in L_{\text {loc }}^{1}\left(\mathbb{R}^{n}\right)$ we define

$$
[u]_{B M O\left(\mathbb{R}^{n}\right)}=\sup _{x_{0} \in \mathbb{R}^{n}, \varrho>0} \frac{1}{\left|B_{\varrho}\left(x_{0}\right)\right|} \int_{B_{\varrho}\left(x_{0}\right)}\left|u(x)-u_{x_{0}, \varrho}\right| d x,
$$

where

$$
u_{x_{0}, \varrho}=\frac{1}{\left|B_{\varrho}\left(x_{0}\right)\right|} \int_{B_{\varrho}\left(x_{0}\right)} u d x .
$$

Then we define the space of functions with bounded mean oscillation as

$$
B M O\left(\mathbb{R}^{n}\right)=\left\{u \in L_{\mathrm{loc}}^{1}\left(\mathbb{R}^{n}\right):[u]_{B M O\left(\mathbb{R}^{n}\right)}<+\infty\right\} .
$$

This space was introduced by John and Nirenberg in [16]. The $B M O$ space is a borderline space, which plays a key role in different areas of Mathematical Analysis, as a natural replacement of $L^{\infty}\left(\mathbb{R}^{n}\right)$ in a large number of results, for instance in interpolation. Fefferman and Stein characterized this space as the dual of the Hardy space $\mathcal{H}^{1}$, see [11, Theorem 1] and [12, Theorem 2]. Another important appearance of this space is in Elliptic Regularity Theory: indeed, the logarithm of a positive local solution to an elliptic partial differential equation is a locally $B M O$ function. This observation is a crucial step in the classical proof by Moser of Harnack's inequality, see [24].

It turns out that the $B M O$ space can be seen as a particular instance of the larger family of Campanato spaces, see [7]. For $1 \leq p<+\infty$ and $0 \leq \lambda \leq n+p$, for every $u \in L_{\text {loc }}^{p}\left(\mathbb{R}^{n}\right)$ we define the seminorm

$$
\begin{gathered}
{[u]_{\mathcal{L}^{p, \lambda}\left(\mathbb{R}^{n}\right)}=\left(\sup _{x_{0} \in \mathbb{R}^{n}, \varrho>0} \varrho^{-\lambda} \int_{B_{\varrho}\left(x_{0}\right)}\left|u-u_{x_{0}, \varrho}\right|^{p} d x\right)^{\frac{1}{p}}} \\
=\sup _{x_{0} \in \mathbb{R}^{n}, \varrho>0} \varrho^{-\lambda / p}\left(\int_{B_{\varrho}\left(x_{0}\right)}\left|u-u_{x_{0}, \varrho}\right|^{p} d x\right)^{\frac{1}{p}} .
\end{gathered}
$$

Accordingly, we introduce the Campanato space

$$
\mathcal{L}^{p, \lambda}\left(\mathbb{R}^{n}\right)=\left\{u \in L_{\mathrm{loc}}^{p}\left(\mathbb{R}^{n}\right):[u]_{\mathcal{L}^{p, \lambda}\left(\mathbb{R}^{n}\right)}<+\infty\right\} .
$$

Notice that since $\left|B_{\varrho}\left(x_{0}\right)\right|=\omega_{n} \varrho^{n}$ we have

$$
[u]_{B M O\left(\mathbb{R}^{n}\right)}=\frac{1}{\omega_{n}}[u]_{\mathcal{L}^{1, n}\left(\mathbb{R}^{n}\right)}, \quad B M O\left(\mathbb{R}^{n}\right)=\mathcal{L}^{1, n}\left(\mathbb{R}^{n}\right) .
$$


We recall that for $n<\lambda \leq n+p$, we have

$$
\frac{1}{C}[u]_{C^{0, \alpha}\left(\mathbb{R}^{n}\right)} \leq[u]_{\mathcal{L}^{p, \lambda}\left(\mathbb{R}^{n}\right)} \leq C[u]_{C^{0, \alpha}\left(\mathbb{R}^{n}\right)}, \quad \text { with } \alpha=\frac{\lambda-n}{p},
$$

see [14, Chapter 2, Section 3]. The constant $C=C(\lambda, n, p)>0$ blows-up as $\lambda \searrow n$.

We must point out that both $[\cdot]_{B M O\left(\mathbb{R}^{n}\right)}$ and $[\cdot]_{\mathcal{L}^{p, \lambda}\left(\mathbb{R}^{n}\right)}$ are only seminorms on their relevant spaces, as they do not detect constants, i.e. the seminorm of constant functions is zero. In order to get a normed space (actually, a Banach space), we need to consider their homogeneous version defined as quotient spaces

$$
B \dot{M} O\left(\mathbb{R}^{n}\right)=\frac{B M O\left(\mathbb{R}^{n}\right)}{\sim_{C}} \quad \text { and } \quad \dot{\mathcal{L}}^{p, \lambda}\left(\mathbb{R}^{n}\right)=\frac{\mathcal{L}^{p, \lambda}\left(\mathbb{R}^{n}\right)}{\sim_{C}},
$$

with the equivalence relation

$$
u \sim_{C} v \Longleftrightarrow u-v \text { is constant almost everywhere. }
$$

We will denote by $\{u\}_{C}$ the class of $u$ with respect to this relation.

An interesting result, which can be found for example in [14, Proposition 2.5 and Corollary 2.3], says that when $\lambda=n$ all the Campanato spaces are isomorphic and we have

$$
B \dot{M} O\left(\mathbb{R}^{n}\right) \simeq \dot{\mathcal{L}}^{p, n}\left(\mathbb{R}^{n}\right), \quad \text { for every } 1 \leq p<+\infty .
$$

On the contrary, for $\lambda \neq n$ the spaces $\mathcal{L}^{p, \lambda}\left(\mathbb{R}^{n}\right)$ and $\mathcal{L}^{q, \lambda}\left(\mathbb{R}^{n}\right)$ do not coincide, for $p \neq q$.

Remark 2.1 As pointed out in [27, Chapter 4, §1.1.1], the definition of the BMO space can be equivalently given by taking hypercubes instead of balls. The same remark applies to Campanato spaces.

\subsection{Weighted integrability for some Campanato spaces}

Functions belonging to $\mathcal{L}^{p, n}\left(\mathbb{R}^{n}\right)$ enjoy a suitable weighted global integrability condition. More precisely, we have the following

Lemma 2.2 Let $1 \leq p<+\infty$ and $R>0$. For every $u \in \mathcal{L}^{p, n}\left(\mathbb{R}^{n}\right)$ such that

$$
\int_{B_{R}(0)} u d x=0
$$

we have

$$
\int_{\mathbb{R}^{n}} \frac{|u|^{p}}{R^{n}+|x|^{n}\left|\log \frac{|x|}{R}\right|^{p+2}} d x \leq C[u]_{\mathcal{L}^{p, n}\left(\mathbb{R}^{n}\right)}^{p},
$$

for some constant $C=C(n, p)>0$.

Proof The proof is an adaptation of that of [12, Equation (1.2)]. The final outcome is slightly better. We observe that it is sufficient to prove (2.3) for $R=1$. The general case then follows by a standard scaling argument.

We first fix some shortcut notation, for the sake of simplicity. For every $k \in \mathbb{N}$, we set

$$
u_{k}=\frac{1}{\left|B_{2^{k}}(0)\right|} \int_{B_{2^{k}}(0)} u d x
$$


and observe that $u_{0}=0$, by assumption. We start by estimating the difference $\left|u_{k+1}-u_{k}\right|$. We have

$$
\begin{aligned}
\left|\int_{B_{2^{k}}(0)}\left[u(x)-u_{k+1}\right] d x\right| & \leq \int_{B_{2^{k}}(0)}\left|u(x)-u_{k+1}\right| d x \\
& \leq\left|B_{2^{k}}(0)\right|^{\frac{p-1}{p}}\left(\int_{B_{2^{k}}(0)}\left|u(x)-u_{k+1}\right|^{p} d x\right)^{\frac{1}{p}} \\
& \leq\left|B_{2^{k}}(0)\right|^{\frac{p-1}{p}}\left(\int_{B_{2^{k+1}}(0)}\left|u(x)-u_{k+1}\right|^{p} d x\right)^{\frac{1}{p}} \\
& \leq C\left|B_{2^{k}}(0)\right|^{\frac{p-1}{p}}\left|B_{2^{k+1}}(0)\right|^{\frac{1}{p}}[u]_{\mathcal{L}^{p, n}\left(\mathbb{R}^{n}\right) .}
\end{aligned}
$$

By observing that

$$
\left(\frac{\left|B_{2^{k+1}}(0)\right|}{\left|B_{2^{k}}(0)\right|}\right)^{\frac{1}{p}}=2^{\frac{n}{p}}
$$

we can divide both sides by $\left|B_{2^{k}}(0)\right|$ and get

$$
\left|u_{k}-u_{k+1}\right| \leq C[u]_{\mathcal{L}^{p, n}}\left(\mathbb{R}^{n}\right) .
$$

By using this estimate and the triangle inequality, we then get for every $j \in \mathbb{N} \backslash\{0\}$

$$
\left|u_{j}\right|=\left|u_{j}-u_{0}\right| \leq \sum_{k=0}^{j-1}\left|u_{k+1}-u_{k}\right| \leq C j[u]_{\mathcal{L}^{p, n}\left(\mathbb{R}^{n}\right)} .
$$

We now get

$$
\begin{aligned}
& \int_{B_{2^{j}}(0)}|u|^{p} d x=\int_{B_{2^{j}}(0)}\left|u-u_{0}\right|^{p} d x \leq 2^{p-1} \int_{B_{2^{j}}(0)}\left|u-u_{j}\right|^{p} d x+2^{p-1}\left|u_{j}\right|^{p}\left|B_{2^{j}}(0)\right| \\
& \quad \leq 2^{p-1} \int_{B_{2^{j}}(0)}\left|u-u_{j}\right|^{p} d x+2^{p-1} C^{p} j^{p}\left|B_{2^{j}}(0)\right|[u]_{\mathcal{L}^{p, n}\left(\mathbb{R}^{n}\right)}^{p} \\
& \leq C\left|B_{2^{j}}(0)\right|\left(1+j^{p}\right)[u]_{\mathcal{L}^{p, n}\left(\mathbb{R}^{n}\right)^{p} .}
\end{aligned}
$$

In particular, this implies for every $j \in \mathbb{N} \backslash\{0\}$

$$
\frac{1}{\left|B_{2^{j}}(0)\right|\left(1+j^{p}\right)} \int_{B_{2^{j}}(0) \backslash B_{2^{j-1}}(0)}|u|^{p} d x \leq C[u]_{\mathcal{L}^{p, n}\left(\mathbb{R}^{n}\right)}^{p} .
$$

We now observe that for every $x \in B_{2^{j}}(0) \backslash B_{2^{j-1}}(0)$

$$
\left|B_{2^{j}}(0)\right|\left(1+j^{p}\right)=\omega_{n} 2^{j n}\left(1+j^{p}\right) \leq \omega_{n}(2|x|)^{n}\left(1+\left(1+\log _{2}|x|\right)^{p}\right) .
$$

If we use this estimate in the previous inequality, we get

$$
\int_{B_{2^{j}}(0) \backslash B_{2^{j-1}}(0)} \frac{|u|^{p}}{1+|x|^{n}(\log |x|)^{p}} d x \leq C[u]_{\mathcal{L}^{p, n}\left(\mathbb{R}^{n}\right)}^{p} .
$$

We further divide both sides by $j^{2}$, so to get

$$
\frac{1}{j^{2}} \int_{B_{2^{j}}(0) \backslash B_{2^{j-1}}(0)} \frac{|u|^{p}}{1+|x|^{n}(\log |x|)^{p}} d x \leq \frac{C}{j^{2}}[u]_{\mathcal{L}^{p, n}\left(\mathbb{R}^{n}\right)}^{p} .
$$


On the left-hand side, we use that for every $x \in B_{2^{j}}(0) \backslash B_{2^{j-1}}(0)$ it holds

$$
j^{2} \leq\left(1+\log _{2}|x|\right)^{2} \leq C\left(1+(\log |x|)^{2}\right)
$$

This in turn implies that

$$
j^{2}\left(1+|x|^{n}(\log |x|)^{p}\right) \leq C\left(1+|x|^{n}(\log |x|)^{p+2}\right),
$$

possibly for a different constant $C>0$. If we now sum over $j \geq 1$ we get

$$
\int_{\mathbb{R}^{n} \backslash B_{1}(0)} \frac{|u|^{p}}{1+|x|^{n}(\log |x|)^{p+2}} d x \leq C[u]_{\mathcal{L}^{p, n}\left(\mathbb{R}^{n}\right)}^{p} .
$$

We are only left with observing that we have (recall that $u$ has average 0 in $B_{1}(0)$ )

$$
\int_{B_{1}(0)}|u|^{p} d x \leq C\left|B_{1}(0)\right|[u]_{\mathcal{L}^{p, n}\left(\mathbb{R}^{n}\right)}^{p},
$$

and

$$
1+|x|^{n}|\log | x||^{p+2} \geq \frac{1}{C}, \quad \text { for } x \in B_{1}(0)
$$

Thus we get

$$
\int_{B_{1}(0)} \frac{|u|^{p}}{1+|x|^{n}|\log | x||^{p+2}} d x \leq C[u]_{\mathcal{L}^{p, n}\left(\mathbb{R}^{n}\right)}^{p},
$$

as well. By summing up (2.4) and (2.5), we get (2.3) for $R=1$, as desired.

Remark 2.3 Due to Eq. (2.2), the previous weighted estimate applies to $B M O$, as well.

\subsection{A Morrey-Campanato-type inequality and applications}

We now prove an inequality relating the Gagliardo-Slobodeckiǔ and Campanato seminorms. This will give us, as corollaries, fractional versions of the Poincaré-Wirtinger and Morrey inequalities.

Theorem 2.4 Let $s \in(0,1)$ and $1 \leq p<+\infty$. Then for every $u \in C_{c}^{\infty}\left(\mathbb{R}^{n}\right)$ we have

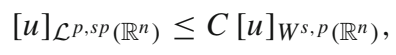

for a constant $C=C(n, p)>0$. 
Proof We fix $x_{0} \in \mathbb{R}^{n}$ and $\varrho>0$, then for every $u, v \in C_{c}^{\infty}\left(\mathbb{R}^{n}\right)$ by Minkowski inequality we get

$$
\begin{aligned}
\left(\varrho^{-s p} \int_{B_{\varrho}\left(x_{0}\right)}\left|u-u_{x_{0}, \varrho}\right|^{p} d x\right)^{\frac{1}{p}} \leq & \left(\varrho^{-s p} \int_{B_{\varrho}\left(x_{0}\right)}|u-v|^{p} d x\right)^{\frac{1}{p}} \\
& +\left(\varrho^{-s p} \int_{B_{\varrho}\left(x_{0}\right)}\left|v-v_{x_{0}, \varrho}\right|^{p} d x\right)^{\frac{1}{p}} \\
& +\left(\varrho^{-s p} \int_{B_{\varrho}\left(x_{0}\right)}\left|v_{x_{0}, \varrho}-u_{x_{0}, \varrho}\right|^{p} d x\right)^{\frac{1}{p}} \\
\leq & 2\left(\varrho^{-s p} \int_{\mathbb{R}^{n}}|u-v|^{p} d x\right)^{\frac{1}{p}} \\
& +\left(\varrho^{-s p} \int_{B_{\varrho}\left(x_{0}\right)}\left|v-v_{x_{0}, \varrho}\right|^{p} d x\right)^{\frac{1}{p}} .
\end{aligned}
$$

In the second estimate, we used Jensen's inequality. We now apply the standard PoincaréWirtinger inequality (see for example [14, Theorem 3.17]) in order to control the last term

$$
\int_{B_{\varrho}\left(x_{0}\right)}\left|v-v_{x_{0}, \varrho}\right|^{p} d x \leq C \varrho^{p} \int_{B_{\varrho}\left(x_{0}\right)}|\nabla v|^{p} d x \leq C \varrho^{p}[v]_{W^{1, p}\left(\mathbb{R}^{n}\right)}^{p},
$$

for a constant $C=C(n, p)>0$. Then the last two displays imply the estimate

$$
\left(\varrho^{-s p} \int_{B_{\varrho}\left(x_{0}\right)}\left|u-u_{x_{0}, \varrho}\right|^{p} d x\right)^{\frac{1}{p}} \leq C \frac{\|u-v\|_{L^{p}\left(\mathbb{R}^{n}\right)}+\varrho[v]_{W^{1, p}\left(\mathbb{R}^{n}\right)}}{\varrho^{s}} .
$$

This estimate is valid for every $v \in C_{c}^{\infty}\left(\mathbb{R}^{n}\right)$. Thus, if we define the $K$-functional

$$
K(t, u):=\inf _{v \in C_{c}^{\infty}\left(\mathbb{R}^{n}\right)}\left(\|u-v\|_{L^{p}\left(\mathbb{R}^{n}\right)}+t[v]_{W^{1, p}\left(\mathbb{R}^{n}\right)}\right),
$$

by taking the infimum over $v$ in the last estimate, we get

$$
\left(\varrho^{-s p} \int_{B_{\varrho}\left(x_{0}\right)}\left|u-u_{x_{0}, \varrho}\right|^{p} d x\right)^{\frac{1}{p}} \leq C \frac{K(\varrho, u)}{\varrho^{s}} .
$$

By raising to power $p$ and integrating over $(0,+\infty)$ with respect to the singular measure $d \varrho / \varrho$, we get

$$
\int_{0}^{+\infty} \varrho^{-s p}\left(\int_{B_{\varrho}\left(x_{0}\right)}\left|u-u_{x_{0}, \varrho}\right|^{p} d x\right) \frac{d \varrho}{\varrho} \leq C \int_{0}^{+\infty}\left(\frac{K(\varrho, u)}{\varrho^{s}}\right)^{p} \frac{d \varrho}{\varrho} .
$$

From [6, Proposition 4.5], we have that

$$
\int_{0}^{+\infty}\left(\frac{K(\varrho, u)}{\varrho^{s}}\right)^{p} \frac{d \varrho}{\varrho} \leq \frac{C}{s(1-s)}[u]_{W^{s, p}\left(\mathbb{R}^{n}\right)}^{p},
$$

for a constant $C=C(n, p)>0$. Up to now, we obtained

$$
\int_{0}^{+\infty} \varrho^{-s p}\left(\int_{B_{\varrho}\left(x_{0}\right)}\left|u-u_{x_{0}, \varrho}\right|^{p} d x\right) \frac{d \varrho}{\varrho} \leq \frac{C}{s(1-s)}[u]_{W^{s, p}\left(\mathbb{R}^{n}\right)}^{p} .
$$


We now use that

$$
\int_{B_{\varrho}\left(x_{0}\right)}\left|u-u_{x_{0}, \varrho}\right|^{p} d x \geq \inf _{c \in \mathbb{R}} \int_{B_{\varrho}\left(x_{0}\right)}|u-c|^{p} d x,
$$

thus we get for $r>0$

$$
\begin{aligned}
\int_{0}^{+\infty} \varrho^{-s p}\left(\int_{B_{\varrho}\left(x_{0}\right)}\left|u-u_{x_{0}, \varrho}\right|^{p} d x\right) \frac{d \varrho}{\varrho} & \geq \int_{r}^{+\infty} \varrho^{-s p}\left(\inf _{c \in \mathbb{R}} \int_{B_{\varrho}\left(x_{0}\right)}|u-c|^{p} d x\right) \frac{d \varrho}{\varrho} \\
& \geq\left(\inf _{c \in \mathbb{R}} \int_{B_{r}\left(x_{0}\right)}|u-c|^{p} d x\right) \int_{r}^{+\infty} \varrho^{-s p} \frac{d \varrho}{\varrho} \\
& =\frac{1}{s p} r^{-s p}\left(\inf _{c \in \mathbb{R}} \int_{B_{r}\left(x_{0}\right)}|u-c|^{p} d x\right) .
\end{aligned}
$$

Since $r>0$ and $x_{0} \in \mathbb{R}^{n}$ are arbitrary, from (2.6) we thus obtain that

$$
\sup _{x_{0} \in \mathbb{R}^{n}, r>0}\left(r^{-s p} \inf _{c \in \mathbb{R}} \int_{B_{r}\left(x_{0}\right)}|u-c|^{p} d x\right) \leq \frac{C}{1-s}[u]_{W^{s, p}\left(\mathbb{R}^{n}\right)}^{p} .
$$

By recalling that the quantity in the left-hand side is equivalent to the Campanato seminorm $\mathcal{L}^{p, s p}$ (see [14, Remark 2.2]), we get

$$
[u]_{\mathcal{L}^{p, s p}\left(\mathbb{R}^{n}\right)}^{p} \leq \frac{C}{1-s}[u]_{W^{s, p}\left(\mathbb{R}^{n}\right)}^{p},
$$

for some $C=C(n, p)>0$. In a similar way, we observe that for $r>0$

$$
\begin{aligned}
\int_{0}^{+\infty} \varrho^{-s p}\left(\int_{B_{\varrho}\left(x_{0}\right)}\left|u-u_{x_{0}, \varrho}\right|^{p} d x\right) \frac{d \varrho}{\varrho} & \geq \int_{r}^{2 r} \varrho^{-s p}\left(\inf _{c \in \mathbb{R}} \int_{B_{\varrho}\left(x_{0}\right)}|u-c|^{p} d x\right) \frac{d \varrho}{\varrho} \\
& \geq\left(\inf _{c \in \mathbb{R}} \int_{B_{r}\left(x_{0}\right)}|u-c|^{p} d x\right) \int_{r}^{2 r} \varrho^{-s p} \frac{d \varrho}{\varrho} \\
& =\left(\inf _{c \in \mathbb{R}} \int_{B_{r}\left(x_{0}\right)}|u-c|^{p} d x\right) \int_{r}^{2 r} \frac{\varrho^{p-s p}}{\varrho^{p}} \frac{d \varrho}{\varrho} \\
& \geq \frac{1}{(2 r)^{p}}\left(\inf _{c \in \mathbb{R}} \int_{B_{r}\left(x_{0}\right)}|u-c|^{p} d x\right) \int_{r}^{2 r} \varrho^{p-s p} \frac{d \varrho}{\varrho} \\
& =\frac{1}{2^{p}(1-s) p} \frac{(2 r)^{p-s p}-r^{p-s p}}{r^{p}} \\
& \left(\inf _{c \in \mathbb{R}} \int_{B_{r}\left(x_{0}\right)}|u-c|^{p} d x\right) .
\end{aligned}
$$

As before, from (2.6) we get

$$
\sup _{x_{0} \in \mathbb{R}^{n}, r>0}\left(r^{-s p} \inf _{c \in \mathbb{R}} \int_{B_{r}\left(x_{0}\right)}|u-c|^{p} d x\right) \leq \frac{C}{s}[u]_{W^{s, p}\left(\mathbb{R}^{n}\right)}^{p},
$$

and thus

$$
[u]_{\mathcal{L}^{p, s p}\left(\mathbb{R}^{n}\right)}^{p} \leq \frac{C}{s}[u]_{W^{s, p}\left(\mathbb{R}^{n}\right)}^{p},
$$

for some $C=C(n, p)>0$. If we now multiply (2.7) by $(1-s),(2.8)$ by $s$ and then take the sum, we get the claimed estimate. 
As a first straightforward consequence of Theorem 2.4, we get the following

Corollary 2.5 (Fractional Poincaré-Wirtinger inequality) Let $s \in(0,1)$ and $1 \leq p<+\infty$. Then for every $u \in C_{c}^{\infty}\left(\mathbb{R}^{n}\right)$ and every $x_{0} \in \mathbb{R}^{n}, R>0$, we have

$$
\int_{B_{R}\left(x_{0}\right)}\left|u-u_{x_{0}, R}\right|^{p} d x \leq C R^{s p}[u]_{W^{s, p}\left(\mathbb{R}^{n}\right)}^{p},
$$

for a constant $C=C(n, p)>0$.

Remark 2.6 As a simple consequence of the previous result, we also have the following more flexible inequality: for every $u \in C_{c}^{\infty}\left(\mathbb{R}^{n}\right)$ and every $x_{0} \in \mathbb{R}^{n}, 0<r \leq R$, we have

$$
\int_{B_{R}\left(x_{0}\right)}\left|u-u_{x_{0}, r}\right|^{p} d x \leq C\left(1+\left(\frac{R}{r}\right)^{n}\right) R^{s p}[u]_{W^{s, p}\left(\mathbb{R}^{n}\right)}^{p},
$$

for a possibly different constant $C=C(n, p)>0$. Indeed, it is sufficient to observe that, due to Jensen's inequality,

$$
\begin{aligned}
& \int_{B_{R}\left(x_{0}\right)}\left|u-u_{x_{0}, r}\right|^{p} d x \leq 2^{p-1} \int_{B_{R}\left(x_{0}\right)}\left|u-u_{x_{0}, R}\right|^{p} d x+2^{p-1}\left|B_{R}\left(x_{0}\right)\right|\left|u_{x_{0}, r}-u_{x_{0}, R}\right|^{p} \\
& \quad \leq 2^{p-1} \int_{B_{R}\left(x_{0}\right)}\left|u-u_{x_{0}, R}\right|^{p} d x+2^{p-1} \frac{\left|B_{R}\left(x_{0}\right)\right|}{\left|B_{r}\left(x_{0}\right)\right|} \int_{B_{r}\left(x_{0}\right)}\left|u-u_{x_{0}, R}\right|^{p} d x \\
& \leq 2^{p-1}\left(1+\left(\frac{R}{r}\right)^{n}\right) \int_{B_{R}\left(x_{0}\right)}\left|u-u_{x_{0}, R}\right|^{p} d x .
\end{aligned}
$$

An application of Corollary 2.5 now leads to the claimed estimate (2.10).

Theorem 2.4, together with the estimate (2.1), also implies the following result. We include in the statement the case $s=1$, which is classical, see for example [14, Theorem 3.9].

Corollary 2.7 (Fractional Morrey's inequality) Let $s \in(0,1]$ and $1 \leq p<+\infty$ be such that $s p>n$. Then for every $u \in C_{c}^{\infty}\left(\mathbb{R}^{n}\right)$ we have

$$
[u]_{C^{0, s-\frac{n}{p}}\left(\mathbb{R}^{n}\right)} \leq C[u]_{W^{s, p}\left(\mathbb{R}^{n}\right)},
$$

for a constant $C=C(n, p, s)>0$. Such a constant may be taken independent of $s$, whenever $s-n / p \geq \delta_{0}$, for some $\delta_{0}>0$. In this case, it has the form $C=C\left(n, p, \delta_{0}\right)>0$.

Remark 2.8 The previous result is well-known, see for example [26, Théorème 8.2] for a proof using a different interpolation-type argument. The main focus here is on the presence of the scaling factor $s(1-s)$, which is incorporated in our definition of the GagliardoSlobodeckiı̌ seminorm. If one is not interested in keeping track of this factor, actually the proof simplifies, see for example [13, Lemma 2.3].

We conclude this section with a variant of the Poincaré-Wirtinger inequality. We do not pay too much attention to the quality of the constant: the resulting outcome will be sufficient for our purposes.

Lemma 2.9 Let $s \in(0,1)$ and $1 \leq p<+\infty$. Let $u \in L_{\mathrm{loc}}^{1}\left(\mathbb{R}^{n}\right)$ be such that

$$
[u]_{W^{s, p}\left(\mathbb{R}^{n}\right)}<+\infty .
$$

The for every $0<r<R$ and $x_{0} \in \mathbb{R}^{n}$, we have

$$
\int_{B_{R}\left(x_{0}\right) \backslash B_{r}\left(x_{0}\right)}\left|u-u_{x_{0}, R}\right|^{p} d x \leq C R^{s p} \iint_{\left(B_{R}\left(x_{0}\right) \backslash B_{r}\left(x_{0}\right)\right) \times B_{R}\left(x_{0}\right)} \frac{|u(x)-u(y)|^{p}}{|x-y|^{n+s p}} d x d y,
$$


for a constant $C=C(n, p, s)>0$.

Proof The proof is quite straightforward, it is the same that can be found in [21, page 297], for example. By using Jensen's inequality, we have

$$
\int_{B_{R}\left(x_{0}\right) \backslash B_{r}\left(x_{0}\right)}\left|u-u_{x_{0}, R}\right|^{p} d x \leq \frac{1}{\left|B_{R}\left(x_{0}\right)\right|} \iint_{\left(B_{R}\left(x_{0}\right) \backslash B_{r}\left(x_{0}\right)\right) \times B_{R}\left(x_{0}\right)}|u(x)-u(y)|^{p} d x d y .
$$

We now observe that

$$
1 \leq \frac{(2 R)^{n+s p}}{|x-y|^{n+s p}}, \quad \text { for a. e. }(x, y) \in B_{R}\left(x_{0}\right) \times B_{R}\left(x_{0}\right) .
$$

By using this simple fact in the previous estimate, we get the desired conclusion.

\section{Characterisation for $s p<n$}

This case is similar to the local case (i.e. $s=1$ ) for $p<n$. Indeed, in this range we obtain an embedding into a Lebesgue space, by means of the fractional Sobolev inequality

$$
\mathcal{S}_{s, p}\|u\|_{L^{p_{s}^{\star}\left(\mathbb{R}^{n}\right)}}^{p} \leq[u]_{W^{s, p}\left(\mathbb{R}^{n}\right)}^{p}, \quad \text { for every } u \in C_{c}^{\infty}\left(\mathbb{R}^{n}\right),
$$

for some constant $\mathcal{S}_{s, p}>0$. Here $p_{s}^{\star}$ is the critical Sobolev exponent, defined by

$$
p_{s}^{\star}=\frac{n p}{n-s p} .
$$

We refer to [19, Theorem 10.2.1] for an elementary proof of (3.1). See also [26, Théorème 8.1] for an older proof, based on real interpolation techniques.

By using inequality (3.1), it is possible to give a concrete characterization of the completion $\mathcal{D}^{s, p}\left(\mathbb{R}^{n}\right)$ as a functional space.

Theorem 3.1 Let $s \in(0,1]$ and $1 \leq p<+\infty$ be such that $s p<n$. We indicate by $\dot{W}^{s, p}\left(\mathbb{R}^{n}\right)$ the space

$$
\dot{W}^{s, p}\left(\mathbb{R}^{n}\right)=\left\{u \in L^{p_{s}^{\star}}\left(\mathbb{R}^{n}\right):[u]_{W^{s, p}\left(\mathbb{R}^{n}\right)}<+\infty\right\} .
$$

We endow this space with the norm

$$
\|u\|_{\dot{W}^{s, p}\left(\mathbb{R}^{n}\right)}=[u]_{W^{s, p}\left(\mathbb{R}^{n}\right)}, \quad \text { for every } u \in \dot{W}^{s, p}\left(\mathbb{R}^{n}\right) .
$$

Then this is a Banach space, having $C_{c}^{\infty}\left(\mathbb{R}^{n}\right)$ as a dense subspace. Moreover, there exists a linear isometric isomorphism

$$
\mathcal{J}: \mathcal{D}^{s, p}\left(\mathbb{R}^{n}\right) \rightarrow \dot{W}^{s, p}\left(\mathbb{R}^{n}\right) .
$$

In other words, the space $\mathcal{D}^{s, p}\left(\mathbb{R}^{n}\right)$ can be identified with $\dot{W}^{s, p}\left(\mathbb{R}^{n}\right)$.

Proof It is easy to see that $\dot{W}^{s, p}\left(\mathbb{R}^{n}\right)$ is a normed vector space. The fact that this is a Banach space will follow from the claimed isometry, that we are going to construct at the end of the proof.

We now divide the rest of the proof in three parts. 
Part 1: density of smooth functions. We prove that $C_{c}^{\infty}\left(\mathbb{R}^{n}\right)$ is dense in $\dot{W}^{s, p}\left(\mathbb{R}^{n}\right)$. We need to prove that for every $u \in \dot{W}^{s, p}\left(\mathbb{R}^{n}\right)$, there exists a sequence $\left(u_{m}\right)_{m \in \mathbb{N}} \subset C_{c}^{\infty}\left(\mathbb{R}^{n}\right)$ such that

$$
\lim _{m \rightarrow \infty}\left[u_{m}-u\right]_{W^{s, p}\left(\mathbb{R}^{n}\right)}=0 .
$$

In order to construct the sequence $\left(u_{m}\right)_{m \in \mathbb{N}}$, we consider the sequence of smoothing kernels $\left(\rho_{m}\right)_{m \geq 1}$ as in the statement of Lemma A.1. Moreover, we introduce a sequence of cut-off functions $\eta_{j} \in C_{c}^{\infty}\left(\mathbb{R}^{n}\right)$ with supp $\eta_{j} \subset B_{2 j}$ such that

$$
0 \leq \eta_{j} \leq 1, \quad \eta_{j} \equiv 1 \text { on } B_{j}, \quad\left|\nabla \eta_{j}\right| \leq \frac{C}{j}
$$

By Lemma B.1, for every $m \geq 1$ we have

$$
\lim _{j \rightarrow \infty}\left[\left(u * \rho_{m}\right) \eta_{j}-u * \rho_{m}\right]_{W^{s, p}\left(\mathbb{R}^{n}\right)}=0 .
$$

Thus, for every $m \geq 1$ we can choose $j_{m} \in \mathbb{N}$ such that

$$
\left[\left(u * \rho_{m}\right) \eta_{j_{m}}-u * \rho_{m}\right]_{W^{s, p}\left(\mathbb{R}^{n}\right)} \leq \frac{1}{m} .
$$

We finally set

$$
u_{m}=\left(u * \rho_{m}\right) \eta_{j_{m}},
$$

then this sequence has the desired approximation property. Indeed, observe that by the triangle inequality we have

$$
\begin{aligned}
{\left[u_{m}-u\right]_{W^{s, p}\left(\mathbb{R}^{n}\right)} } & =\left[\left(u * \rho_{m}\right) \eta_{j_{m}}-u\right]_{W^{s, p}\left(\mathbb{R}^{n}\right)} \\
& \leq\left[\left(u * \rho_{m}\right) \eta_{j_{m}}-u * \rho_{m}\right]_{W^{s, p}\left(\mathbb{R}^{n}\right)}+\left[u * \rho_{m}-u\right]_{W^{s, p}\left(\mathbb{R}^{n}\right)} \\
& \leq \frac{1}{m}+\left[u * \rho_{m}-u\right]_{W^{s, p}\left(\mathbb{R}^{n}\right) .}
\end{aligned}
$$

By taking the limit as $m$ goes to $\infty$ and appealing to Lemma A.1, we get the conclusion.

Part 2: Cauchy sequences in $\mathcal{D}^{s, p}\left(\mathbb{R}^{n}\right)$. We take a sequence $\left(u_{m}\right)_{m \in \mathbb{N}} \subset C_{c}^{\infty}\left(\mathbb{R}^{n}\right)$, which is a Cauchy sequence with respect to the norm

$$
\varphi \mapsto[\varphi]_{W^{s, p}\left(\mathbb{R}^{n}\right)} .
$$

By using the fractional Sobolev inequality (3.1), we have that this is a Cauchy sequence in $L^{p_{s}^{\star}}\left(\mathbb{R}^{n}\right)$, as well. The latter being a Banach space, we get that the sequence converges strongly in $L^{p_{s}^{\star}}\left(\mathbb{R}^{n}\right)$ to a function $u \in L^{p_{s}^{\star}}\left(\mathbb{R}^{n}\right)$. Furthermore, we can show that $u \in \dot{W}^{s, p}\left(\mathbb{R}^{n}\right)$.

Indeed, if we fix $\varepsilon>0$, then by definition of Cauchy sequence there exists $n_{\varepsilon} \in \mathbb{N}$ such that

$$
\left[u_{m}-u_{k}\right]_{W^{s, p}\left(\mathbb{R}^{n}\right)}<\varepsilon, \quad \text { for every } k, m \geq n_{\varepsilon} .
$$

In particular, by Minkowski inequality we get

$$
\begin{aligned}
& {\left[u_{m}\right]_{W^{s, p}\left(\mathbb{R}^{n}\right)} \leq\left[u_{m}-u_{n_{\varepsilon}}\right]_{W^{s, p}\left(\mathbb{R}^{n}\right)}+\left[u_{n_{\varepsilon}}\right]_{W^{s, p}\left(\mathbb{R}^{n}\right)} } \\
&<\varepsilon+\left[u_{n_{\varepsilon}}\right]_{W^{s, p}\left(\mathbb{R}^{n}\right)}, \quad \text { for every } m \geq n_{\varepsilon} .
\end{aligned}
$$

This shows that the sequence

$$
\left(\frac{u_{m}(x)-u_{m}(y)}{|x-y|^{\frac{n}{p}+s}}\right)_{m \in \mathbb{N}} \subset L^{p}\left(\mathbb{R}^{n} \times \mathbb{R}^{n}\right),
$$


is bounded in $L^{p}\left(\mathbb{R}^{n} \times \mathbb{R}^{n}\right)$. By using that $u_{m}$ converges almost everywhere to $u$ (up to a subsequence), Fatou's Lemma entails

$$
[u]_{W^{s, p}\left(\mathbb{R}^{n}\right)} \leq \liminf _{m \rightarrow \infty}\left[u_{m}\right]_{W^{s, p}\left(\mathbb{R}^{n}\right)}<+\infty,
$$

i.e. $u \in \dot{W}^{s, p}\left(\mathbb{R}^{n}\right)$.

We now observe that $L^{p}\left(\mathbb{R}^{n} \times \mathbb{R}^{n}\right)$ is a Banach space, thus the Cauchy sequence (3.3) converges strongly in $L^{p}\left(\mathbb{R}^{n} \times \mathbb{R}^{n}\right)$. By uniqueness, the limit must coincide with

$$
\frac{u(x)-u(y)}{|x-y|^{\frac{n}{p}+s}} \text {. }
$$

In conclusion, we obtain that the Cauchy sequence $\left(u_{m}\right)_{m \in \mathbb{N}}$ converges with respect to the Gagliardo-Slobodeckiǔ seminorm to an element of $\dot{W}^{s, p}\left(\mathbb{R}^{n}\right)$.

Part 3: construction of the isometry. We now take $U \in \mathcal{D}^{s, p}\left(\mathbb{R}^{n}\right)$ and choose a representative of this equivalence class, i.e. $U=\left\{\left(u_{m}\right)_{m \in \mathbb{N}}\right\}_{s, p}$. Thanks to Part 2, we know that $\left(u_{m}\right)_{m \in \mathbb{N}}$ converges to a function $u \in \dot{W}^{s, p}\left(\mathbb{R}^{n}\right)$. We then define

$$
\mathcal{J}(U)=u \text {. }
$$

Observe that this is well-defined, since for any other representative $\left(\tilde{u}_{m}\right)_{m \in \mathbb{N}}$ belonging to the class $U$, we still have

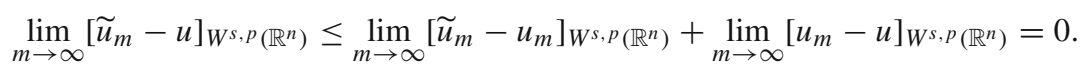

Moreover, it is easy to see that $\mathcal{J}$ is linear. It is also immediate to obtain that this is an isometry, since

$$
\|U\|_{\mathcal{D}^{s, p}\left(\mathbb{R}^{n}\right)}=\lim _{m \rightarrow \infty}\left[u_{m}\right]_{W^{s, p}\left(\mathbb{R}^{n}\right)}=[u]_{W^{s, p}\left(\mathbb{R}^{n}\right)}=\|u\|_{\dot{W}^{s, p}}=\|\mathcal{J}(U)\|_{\dot{W}^{s, p}} .
$$

We are left with proving that $\mathcal{J}$ is surjective. From Part 1 we know that for every $u \in$ $\dot{W}^{s, p}\left(\mathbb{R}^{n}\right)$ there exists a sequence $\left(u_{m}\right)_{n \in \mathbb{N}} \subset C_{c}^{\infty}\left(\mathbb{R}^{n}\right)$ such that

$$
\lim _{n \rightarrow \infty}\left[u_{m}-u\right]_{W^{s, p}\left(\mathbb{R}^{n}\right)}=0 .
$$

In particular, this is a Cauchy sequence with respect to the Gagliardo-Slobodeckir seminorm. Thus we get

$$
u=\mathcal{J}\left(\left\{\left(u_{m}\right)_{m \in \mathbb{N}}\right\}_{s, p}\right)
$$

This concludes the proof.

Remark 3.2 We note that

$$
W^{s, p}\left(\mathbb{R}^{n}\right) \subset L^{p}\left(\mathbb{R}^{n}\right) \cap L^{p_{s}^{\star}}\left(\mathbb{R}^{n}\right),
$$

with continuous inclusion. The inclusion in $L^{p}\left(\mathbb{R}^{n}\right)$ is a straightforward consequence of the definition (1.1) of $W^{s, p}\left(\mathbb{R}^{n}\right)$. On the other hand, the inclusion in $L^{p_{s}^{\star}}\left(\mathbb{R}^{n}\right)$ follows from the fractional Sobolev inequality and the density of $C_{c}^{\infty}\left(\mathbb{R}^{n}\right)$ functions in $W^{s, p}\left(\mathbb{R}^{n}\right)$.

We can exploit this summability information to see that

$$
W^{s, p}\left(\mathbb{R}^{n}\right) \subsetneq \dot{W}^{s, p}\left(\mathbb{R}^{n}\right) .
$$

For example, it is not difficult to see that the function

$$
\varphi(x)=\left(1+|x|^{2}\right)^{-\frac{\alpha}{2}}, \quad \text { for } \frac{n}{p}-s<\alpha \leq \frac{n}{p},
$$


is such that

$$
\varphi \in \dot{W}^{s, p}\left(\mathbb{R}^{n}\right) \backslash W^{s, p}\left(\mathbb{R}^{n}\right) .
$$

Indeed, $\varphi \notin L^{p}\left(\mathbb{R}^{n}\right)$ thanks to the choice of $\alpha$. In order to see that $\varphi$ has a finite GagliardoSlobodeckir seminorm, it is useful to decompose the seminorm as follows

$$
\begin{aligned}
\frac{1}{s(1-s)}[\varphi]_{W^{s, p}\left(\mathbb{R}^{n}\right)}^{p}= & \iint_{\left(\mathbb{R}^{n} \backslash B_{1}(0)\right) \times\left(\mathbb{R}^{n} \backslash B_{1}(0)\right)} \frac{|\varphi(x)-\varphi(y)|^{p}}{|x-y|^{n+s p}} d x d y \\
& +\iint_{B_{1}(0) \times B_{1}(0)} \frac{|\varphi(x)-\varphi(y)|^{p}}{|x-y|^{n+s p}} d x d y \\
& +2 \iint_{B_{1}(0) \times\left(\mathbb{R}^{n} \backslash B_{2}(0)\right)} \frac{|\varphi(x)-\varphi(y)|^{p}}{|x-y|^{n+s} p} d x d y \\
& +2 \iint_{B_{1}(0) \times\left(B_{2}(0) \backslash B_{1}(0)\right)} \frac{|\varphi(x)-\varphi(y)|^{p}}{|x-y|^{n+s p}} d x d y=: \mathcal{I}_{1}+\mathcal{I}_{2}+\mathcal{I}_{3}+\mathcal{I}_{4} .
\end{aligned}
$$

The integrals $\mathcal{I}_{2}$ and $\mathcal{I}_{4}$ are finite, thanks to the Lipschitz character of $\varphi$. The third integral $\mathcal{I}_{3}$ is finite, by using that $\varphi \in L^{\infty}\left(\mathbb{R}^{n}\right)$ and that the function

$$
x \mapsto \int_{\mathbb{R}^{n} \backslash B_{2}(0)} \frac{1}{|x-y|^{n+s p}} d y,
$$

is uniformly bounded for $x \in B_{1}(0)$. Finally, for the finiteness of $\mathcal{I}_{1}$, it is sufficient to observe that ${ }^{1}$

$$
|\varphi(x)-\varphi(y)| \leq\left. C|| x\right|^{-\alpha}-|y|^{-\alpha} \mid, \quad \text { for every } x, y \in \mathbb{R}^{n} \backslash B_{1}(0),
$$

so that

$$
\mathcal{I}_{1} \leq \iint_{\left(\mathbb{R}^{n} \backslash B_{1}(0)\right) \times\left(\mathbb{R}^{n} \backslash B_{1}(0)\right)} \frac{|\Psi(x)-\Psi(y)|^{p}}{|x-y|^{n+s p}} d x d y, \quad \text { with } \Psi(x)=|x|^{-\alpha} .
$$

The last double integral is then finite, by appealing to [5, Lemma A.1].

Remark 3.3 We take the occasion to recall that the interesting question of determining the sharp constant in (3.1) is still open, except for the case $p=2$, solved in [8]. It is clear that the sharp constant is given by

$$
\mathcal{S}_{s, p}=\inf _{u \in \mathcal{D}^{s, p}\left(\mathbb{R}^{n}\right)}\left\{[u]_{W^{s, p}\left(\mathbb{R}^{n}\right)}^{p}:\|u\|_{L^{p_{s}^{\star}}\left(\mathbb{R}^{n}\right)}=1\right\} .
$$

The relevant Euler-Lagrange optimality condition is a nonlinear eigenvalue-type equation involving the operator $\left(-\Delta_{p}\right)^{s}$, already presented in the Introduction. Namely, an extremal for the previous problem has to be a constant sign solution of

$$
\left(-\Delta_{p}\right)^{s} u=\mathcal{S}_{s, p} u^{p_{s}^{\star}-1}, \quad \text { in } \mathbb{R}^{n} .
$$

${ }^{1}$ We can use the following fact: if we set

$$
f(\tau)=\frac{\tau}{\left(1+\tau^{\frac{2}{\alpha}}\right)^{\frac{\alpha}{2}}}, \quad \text { for } \tau \geq 0,
$$

then this is a Lipschitz function and we have

$$
\varphi(x)=\left(1+|x|^{2}\right)^{-\frac{\alpha}{2}}=f\left(|x|^{-\alpha}\right) .
$$


Some properties of solutions to (3.4) have been investigated in [5, Theorem 1.1].

\section{Characterisation for $s p>n$}

Instead of the fractional Sobolev inequality, in this range we have the fractional Morrey inequality, see Corollary 2.7. However, unlike Sobolev's inequality, inequality (2.11) does not detect constants. Even worse, in this range constant functions can be approximated by sequences in $C_{c}^{\infty}\left(\mathbb{R}^{n}\right)$ with respect to the Gagliardo-Slobodeckil seminorm.

Lemma 4.1 Let $s \in(0,1]$ and $s p>n$. There exists a sequence $\left(\varphi_{m}\right)_{m \in \mathbb{N}} \subset C_{c}^{\infty}\left(\mathbb{R}^{n}\right)$ such that

$$
\left[\varphi_{m}\right]_{W^{s, p}\left(\mathbb{R}^{n}\right)} \leq C m^{\frac{n}{p}-s} \rightarrow 0 \text { and } \varphi_{m} \rightarrow 1 \text { uniformly over compact sets, }
$$

as $m \rightarrow+\infty$. Hence, $\left(\varphi_{m}\right)_{m \in \mathbb{N}}$ is equivalent in $\mathcal{D}^{s, p}\left(\mathbb{R}^{n}\right)$ to zero, although its pointwise limit is 1 .

Proof The proof is just based on the scaling properties of the Sobolev-Slobodeckiř seminorm, as in the local case. Let $\varphi \in C_{c}^{\infty}\left(B_{2}\right)$ be a non-negative cut-off function, such that $\varphi$ coincides identically with 1 on $B_{1}$. We define the rescaled sequence

$$
\varphi_{m}(x)=\varphi\left(\frac{x}{m}\right), \quad \text { for every } m \geq 1
$$

By recalling (1.3), we have that

$$
\left[\varphi_{m}\right]_{W^{s, p}\left(\mathbb{R}^{n}\right)}=m^{\frac{n}{p}-s}[\varphi]_{W^{s, p}\left(\mathbb{R}^{n}\right)} .
$$

The conclusion now follows, thanks to the fact that $n / p-s<0$.

Remark 4.2 Notice that this construction is linked with the relative $(s, p)$-capacity of a set $\omega \Subset \Omega$, defined as

$$
(s, p)-\operatorname{cap}_{\Omega}(\omega)=\inf \left\{\iint_{\Omega \times \Omega} \frac{|\varphi(x)-\varphi(y)|^{p}}{|x-y|^{n+s p}} d x d y: \varphi \in C_{c}^{\infty}(\Omega), \varphi=1 \text { in } \omega\right\},
$$

see for example [33]. For $s=1$ this value can be explicitly computed, and the relevant Euler-Lagrange equation in linked to the usual $p$-Laplacian.

We need the following technical result.

Lemma 4.3 Let $s \in(0,1]$ and $s p>n$, we define $\alpha=s-n / p$. Let $\left(u_{m}\right)_{m \in \mathbb{N}} \subset C_{c}^{\infty}\left(\mathbb{R}^{n}\right)$ be a

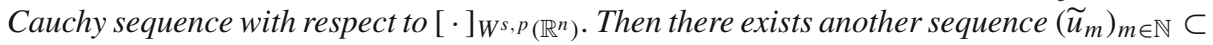
$C_{c}^{\infty}\left(\mathbb{R}^{n}\right)$ such that:

- we have

$$
\lim _{m \rightarrow \infty}\left[u_{m}-\widetilde{u}_{m}\right]_{W^{s, p}\left(\mathbb{R}^{n}\right)}=0,
$$

- $\tilde{u}_{m}$ converges uniformly over compact sets to a $\alpha$-Hölder continuous function $u$.

Moreover, this function $u$ is such that $u(0)=0$,

$$
\left[\tilde{u}_{m}-u\right]_{W^{s, p}\left(\mathbb{R}^{n}\right)} \rightarrow 0, \quad[u]_{W^{s, p}\left(\mathbb{R}^{n}\right)}=\lim _{m \rightarrow \infty}\left[u_{m}\right]_{W^{s, p}\left(\mathbb{R}^{n}\right)},
$$

and

$$
|u(x)-u(y)| \leq C[u]_{W^{s, p}\left(\mathbb{R}^{n}\right)}|x-y|^{\alpha}, \quad \text { for every } x, y \in \mathbb{R}^{n} .
$$


Proof We construct the sequence as follows. We take

$$
M_{m} \geq \max \left\{m,\left(m\left|u_{m}(0)\right|\right)^{\frac{p}{s p-n}}\right\},
$$

and consider $\varphi_{m}$ given by Lemma 4.1. By construction $M_{m}$ diverges to $\rightarrow+\infty$ as $m$ goes to $+\infty$. Then we define

$$
\tilde{u}_{m}(x)=u_{m}(x)-u_{m}(0) \varphi_{M_{m}}(x), \quad \text { for } m \in \mathbb{N} .
$$

We now show that $\tilde{u}_{m}$ has the claimed properties. At first, by construction we have

$$
\begin{aligned}
{\left[u_{m}-\widetilde{u}_{m}\right]_{W^{s, p}\left(\mathbb{R}^{n}\right)}=\left|u_{m}(0)\right|\left[\varphi_{M_{m}}\right]_{W^{s, p}\left(\mathbb{R}^{n}\right)} } & \leq C\left|u_{m}(0)\right| M_{m}^{\frac{n-s p}{p}} \\
& \leq C\left|u_{m}(0)\right|\left(m\left|u_{m}(0)\right|\right)^{-1},
\end{aligned}
$$

which implies that

$$
\lim _{m \rightarrow \infty}\left[u_{m}-\tilde{u}_{m}\right]_{W^{s, p}\left(\mathbb{R}^{n}\right)}=0,
$$

as desired. Observe that this implies that $\left(\widetilde{u}_{m}\right)_{m \in \mathbb{N}} \subset C_{c}^{\infty}\left(\mathbb{R}^{n}\right)$ is still a Cauchy sequence with respect to $[\cdot]_{W^{s, p}}\left(\mathbb{R}^{n}\right)$.

In order to infer the uniform convergence, it is sufficient to show that $\left(\widetilde{u}_{m}\right)_{m \in \mathbb{N}}$ is a Cauchy sequence in $C(K)$, for every $K \subset \mathbb{R}^{n}$ compact set. This follows directly, by applying (2.11) to $\widetilde{u}_{m}-\widetilde{u}_{k}$, i.e.

$$
\left|\tilde{u}_{m}(x)-\tilde{u}_{k}(x)\right| \leq C|x|^{\alpha}\left[\tilde{u}_{m}-\tilde{u}_{k}\right]_{W^{s, p}\left(\mathbb{R}^{n}\right)} .
$$

Hence, it converges uniformly to some function $u \in C(K)$. Since $K$ is arbitrary and the limit is unique, $u$ is defined for every $x \in \mathbb{R}^{n}$. Moreover, it holds $u(0)=0$, since we have $\tilde{u}_{m}(0)=0$ by construction.

By using that $\left(\widetilde{u}_{m}\right)_{m \in \mathbb{N}} \subset C_{c}^{\infty}\left(\mathbb{R}^{n}\right)$ is still a Cauchy sequence with respect to $[\cdot]_{W^{s, p}}\left(\mathbb{R}^{n}\right)$ and arguing as in Part 2 of the proof of Theorem 3.1, we deduce (4.1). The estimate (4.2) can then be obtained by passing to the limit in (2.11).

The major difference with respect to the case $s p<n$ is that now the elements in $\mathcal{D}^{s, p}\left(\mathbb{R}^{n}\right)$ can not be uniquely represented by functions. Indeed, when $s p>n$, any sequence $\left(u_{m}\right)_{m \in \mathbb{N}} \subset C_{c}^{\infty}\left(\mathbb{R}^{n}\right)$ which is Cauchy in the norm $[\cdot]_{W^{s, p}\left(\mathbb{R}^{n}\right)}$ is equivalent to the sequence

$$
v_{m}=u_{m}+C \varphi_{m},
$$

for any constant $C \in \mathbb{R}$. Here $\varphi_{m}$ is the same as in Lemma 4.1.

However, one can show that functions that are approximated by equivalent Cauchy sequences, actually coincide up to a constant. In other words, the homogeneous space $\mathcal{D}^{s, p}\left(\mathbb{R}^{n}\right)$ can be identified with a space of equivalence classes of functions differing by an additive constant.

More precisely, we have the following characterization, which is the main result of this section.

Theorem 4.4 Let $s \in(0,1]$ and $s p>n$, we set $\alpha=s-n / p$. We consider the quotient space

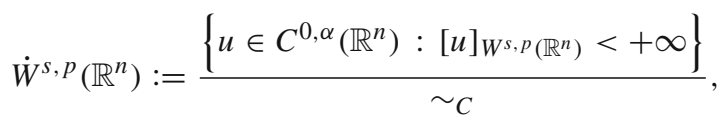


where

$$
u \sim_{C} v \Longleftrightarrow u-v \text { is constant }
$$

We will denote by $\{u\}_{C}$ the equivalence class of $u$ with respect to this relation. We endow this space with the norm

$$
\left\|\{u\}_{C}\right\|_{\dot{W}^{s, p}\left(\mathbb{R}^{n}\right)}=[u]_{W^{s, p}\left(\mathbb{R}^{n}\right)}, \quad \text { for every } u \in C^{0, \alpha}\left(\mathbb{R}^{n}\right) \text { such that }[u]_{W^{s, p}\left(\mathbb{R}^{n}\right)}<+\infty .
$$

Then this is a Banach space and there exists a linear isometric isomorphism

$$
\mathcal{J}: \mathcal{D}^{s, p}\left(\mathbb{R}^{n}\right) \rightarrow \dot{W}^{s, p}\left(\mathbb{R}^{n}\right) .
$$

In other words, the space $\mathcal{D}^{s, p}\left(\mathbb{R}^{n}\right)$ can be identified with $\dot{W}^{s, p}\left(\mathbb{R}^{n}\right)$.

Proof It is immediate to see that $\dot{W}^{s, p}\left(\mathbb{R}^{n}\right)$ is a normed vector space. Indeed, constant functions all belong to the equivalence class $\{0\}_{C}$.

We now construct the isometry. The fact that $\dot{W}^{s, p}\left(\mathbb{R}^{n}\right)$ is a Banach space will follow at once. For any class

$$
U=\left\{\left(u_{m}\right)_{m \in \mathbb{N}}\right\}_{s, p} \in \mathcal{D}^{s, p}\left(\mathbb{R}^{n}\right),
$$

we may apply Lemma 4.3 and consider the new Cauchy sequence $\left(\widetilde{u}_{m}\right)_{m \in \mathbb{N}}$. By construction, we have

$$
U=\left\{\left(u_{m}\right)_{m \in \mathbb{N}}\right\}_{s, p}=\left\{\left(\tilde{u}_{m}\right)_{m \in \mathbb{N}}\right\}_{s, p},
$$

and by Lemma 4.3 , we know that $\left(\tilde{u}_{m}\right)_{m \in \mathbb{N}}$ converges to some function

$$
u \in\left\{\varphi \in C^{0, \alpha}\left(\mathbb{R}^{n}\right):[\varphi]_{W^{s, p}\left(\mathbb{R}^{n}\right)}<+\infty\right\} .
$$

We may identify $U$ with the equivalence class $\{u\}_{C}$, i. e. we define $\mathcal{J}(U)=\{u\}_{C}$.

Observe that this is well-defined, since for any other representative $\left(v_{m}\right)_{m \in \mathbb{N}}$ belonging to the class $U$, we still have

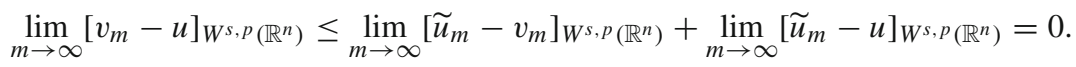

Let us show that the map $\mathcal{J}$ is linear. Indeed for every $U, V \in \mathcal{D}^{s, p}\left(\mathbb{R}^{n}\right)$ and $\alpha, \beta \in$ $\mathbb{R}$, we consider $\alpha U+\beta V$. By choosing $\left(u_{m}\right)_{m \in \mathbb{N}}$ a representative for $U$ and $\left(v_{m}\right)_{m \in \mathbb{N}}$ a representative for $V$, we can apply Lemma 4.3 to both sequences and obtain two new sequences $\left(\widetilde{u}_{m}\right)_{m \in \mathbb{N}},\left(\widetilde{v}_{m}\right)_{m \in \mathbb{N}}$

$$
\left(\tilde{u}_{m}\right)_{m \in \mathbb{N}} \sim_{s, p}\left(u_{m}\right)_{m \in \mathbb{N}} \text { and }\left(\widetilde{v}_{m}\right)_{m \in \mathbb{N}} \sim_{s, p}\left(v_{m}\right)_{m \in \mathbb{N}},
$$

and two functions

$$
u, v \in\left\{\varphi \in C^{0, \alpha}\left(\mathbb{R}^{n}\right):[\varphi]_{W^{s, p}\left(\mathbb{R}^{n}\right)}<+\infty\right\},
$$

such that we have

$$
\mathcal{J}(U)=\{u\}_{C} \quad \text { and } \quad \mathcal{J}(V)=\{v\}_{C} .
$$

By observing that

$$
\alpha U+\beta V=\left\{\left(\alpha \tilde{u}_{m}+\beta \widetilde{v}_{m}\right)\right\}_{s, p},
$$

and using that

$$
\lim _{m \rightarrow \infty}\left[\left(\alpha \tilde{u}_{m}+\beta \widetilde{v}_{m}\right)-(\alpha u+\beta v)\right]_{W^{s, p}\left(\mathbb{R}^{n}\right)}=0,
$$


we get

$$
\mathcal{J}(\alpha U+\beta V)=\{\alpha u+\beta v\}_{C}=\alpha\{u\}_{C}+\beta\{v\}_{C}=\alpha \mathcal{J}(U)+\beta \mathcal{J}(V),
$$

as desired.

Moreover, by construction we have

$$
\|\mathcal{J}(U)\|_{\dot{W}^{s, p}\left(\mathbb{R}^{n}\right)}=\left\|\{u\}_{C}\right\|_{\dot{W}^{s, p}\left(\mathbb{R}^{n}\right)}=[u]_{W^{s, p}\left(\mathbb{R}^{n}\right)}=\lim _{m \rightarrow \infty}\left[\widetilde{u}_{m}\right]_{W^{s, p}\left(\mathbb{R}^{n}\right)}=\|U\|_{\mathcal{D}^{s, p}\left(\mathbb{R}^{n}\right)},
$$

which implies that this is an isometry.

We still have to show that $\mathcal{J}$ is surjective. For every equivalence class $\{v\}_{C} \in \dot{W}^{s, p}\left(\mathbb{R}^{n}\right)$, we may select the representative $v$ in such a way that

$$
v(0)=0 .
$$

Then we can construct a sequence $\left(v_{m}\right)_{m \in \mathbb{N}} \subset C_{c}^{\infty}\left(\mathbb{R}^{n}\right)$ such that

$$
\lim _{m \rightarrow \infty}\left[v_{m}-v\right]_{W^{s, p}\left(\mathbb{R}^{n}\right)}=0 .
$$

In order to do this, we can repeat the construction of Part 1 in the proof of Theorem 3.1, up to some modifications that we are going to detail. More precisely, we introduce a sequence of cut-off functions $\eta_{j} \in C_{c}^{\infty}\left(\mathbb{R}^{n}\right)$ with supp $\eta_{j} \subset B_{2 j}(0)$ such that

$$
0 \leq \eta_{j} \leq 1, \quad \eta_{j} \equiv 1 \text { on } B_{j}(0), \quad\left|\nabla \eta_{j}\right| \leq \frac{C}{j},
$$

and observe that by Lemma B.2, for every $m \geq 1$

$$
\left[\left(u * \rho_{m}\right) \eta_{j}-u * \rho_{m}\right]_{W^{s, p}\left(\mathbb{R}^{n}\right)} \leq C,
$$

for a constant $C>0$ independent of $j$. This shows that the sequence

$$
\left(\frac{\left(u * \rho_{m}(x)\right)\left(\eta_{j}(x)-1\right)-\left(u * \rho_{m}(y)\right)\left(\eta_{j}(y)-1\right)}{|x-y|^{\frac{n}{p}+s}}\right)_{j \in \mathbb{N}} \subset L^{p}\left(\mathbb{R}^{n} \times \mathbb{R}^{n}\right),
$$

weakly converges, up to a subsequence. The weak limit is given by the null function, since $1-\eta_{j}$ converges to 0 , locally uniformly. In order to upgrade this convergence, we can apply Mazur's Lemma to infer that there exists a new sequence made of convex combinations of (4.4), which converges strongly to 0 in $L^{p}\left(\mathbb{R}^{n} \times \mathbb{R}^{n}\right)$.

Thanks to the form of the sequence (4.4), this finally implies that there exists $\left(\tilde{\eta}_{j}\right)_{j \in \mathbb{N}} \subset$ $C_{c}^{\infty}\left(\mathbb{R}^{n}\right)$, such that

$$
\lim _{j \rightarrow \infty}\left\|\frac{\left(u * \rho_{m}(x)\right)\left(\tilde{\eta}_{j}(x)-1\right)-\left(u * \rho_{m}(y)\right)\left(\tilde{\eta}_{j}(y)-1\right)}{|x-y|^{\frac{n}{p}+s}}\right\|_{L^{p}\left(\mathbb{R}^{n} \times \mathbb{R}^{n}\right)}=0 .
$$

in other words, we have

$$
\lim _{j \rightarrow \infty}\left[\left(u * \rho_{m}\right) \tilde{\eta}_{j}-u * \rho_{m}\right]_{W^{s, p}\left(\mathbb{R}^{n}\right)}=0 .
$$

Thus, for every $m \geq 1$ we can choose $j_{m} \in \mathbb{N}$ such that

$$
\left[\left(u * \rho_{m}\right) \tilde{\eta}_{j_{m}}-u * \rho_{m}\right]_{W^{s, p}\left(\mathbb{R}^{n}\right)} \leq \frac{1}{m} .
$$

If we now define the sequence $\left(v_{m}\right)_{m \in \mathbb{N}} \subset C_{c}^{\infty}\left(\mathbb{R}^{n}\right)$ by

$$
v_{m}=\left(u * \rho_{m}\right) \tilde{\eta}_{j_{m}},
$$


it is easy to see that this verifies (4.3), thanks to the choice of $j_{m}$ and Lemma A.1.

Thus we can identify $\{v\}_{C}$ with the equivalence class $\left\{\left(v_{m}\right)_{m \in \mathbb{N}}\right\}_{s, p}$. In other words, this proves the surjectivity of $\mathcal{J}$. The proof is over.

Remark 4.5 We recall that we indicate

$$
C^{0, \alpha}\left(\mathbb{R}^{n}\right)=\left\{u: \mathbb{R}^{n} \rightarrow \mathbb{R}: \sup _{x \neq y} \frac{|u(x)-u(y)|}{|x-y|^{\alpha}}<+\infty\right\},
$$

thus the functions belonging to this space are not necessarily bounded.

\section{Characterisation for $s p=n$}

\subsection{General case}

We start with the corresponding version Lemma 4.1 for the case $s p=n$. The construction now is slightly more complicated, in particular it cannot be an easy consequence of scalings, as we have explained in the Introduction. Hence, a careful choice of auxiliary functions is needed.

Lemma 5.1 Let $s \in(0,1]$ and $n \geq 1$ be such that $s<n$. There exists a sequence $\left(\varphi_{m}\right)_{m \in \mathbb{N}} \subset$ $C_{c}^{\infty}\left(\mathbb{R}^{n}\right)$ such that

$$
\left[\varphi_{m}\right]_{W^{s, \frac{n}{s}\left(\mathbb{R}^{n}\right)}} \leq C\left(\frac{1}{\log m}\right)^{1-\frac{s}{n}} \rightarrow 0 \quad \text { and } \quad \varphi_{m} \rightarrow 1 \text { uniformly over compact sets }
$$

as $m \rightarrow+\infty$. Hence, $\left(\varphi_{m}\right)_{m \in \mathbb{N}}$ is equivalent in $\mathcal{D}^{s, \frac{n}{s}}\left(\mathbb{R}^{n}\right)$ to zero, although its pointwise limit is 1 .

Proof As in [10, page 319] and [19, Lemma 15.2.2], we take the sequence

$$
\psi_{m}(x)= \begin{cases}1, & \text { if }|x|<m, \\ \frac{1}{\log m} \log \frac{m^{2}}{|x|}, & \text { if } m \leq|x| \leq m^{2}, \\ 0, & \text { if }|x|>m^{2} .\end{cases}
$$

For $s=1$, it can be checked by direct computation that

$$
\lim _{m \rightarrow \infty}\left\|\nabla \psi_{m}\right\|_{L^{n}\left(\mathbb{R}^{n}\right)}=0 .
$$

For the fractional case $s \in(0,1)$, we claim that we still have

$$
\lim _{m \rightarrow \infty}\left[\psi_{m}\right]_{W^{s, \frac{n}{s}}\left(\mathbb{R}^{n}\right)}=0
$$


but the direct computation is fairly more intricate. We introduce the sequence

$$
u_{m}(x)= \begin{cases}\left|\log \frac{1}{m}\right|^{\frac{n-s}{n}}, & \text { if }|x|<\frac{1}{m}, \\ \left|\log \frac{1}{m}\right|^{-\frac{s}{n}} \log |x|, & \text { if } \frac{1}{m} \leq|x| \leq 1, \\ 0, & \text { if }|x|>1,\end{cases}
$$

and observe that this is related to $\psi_{m}$ through the relation

$$
\psi_{m}(x)=\left|\log \frac{1}{m}\right|^{\frac{s-n}{n}} u_{m}\left(\frac{x}{m^{2}}\right) .
$$

By using that the Gagliardo-Slobodeckiľ seminorm is now scale invariant, we thus get

$$
\left[\psi_{m}\right]_{W^{s, \frac{n}{s}}\left(\mathbb{R}^{n}\right)}=\left|\log \frac{1}{m}\right|^{\frac{s-n}{n}}\left[u_{m}\right]_{W^{s, \frac{n}{s}}\left(\mathbb{R}^{n}\right)} .
$$

We now recall that from [25, Proposition 5.1] we have

$$
\lim _{m \rightarrow \infty}\left[u_{m}\right]_{W^{s, \frac{n}{s}}\left(\mathbb{R}^{n}\right)}=\gamma_{n, s}<+\infty .
$$

We finally get that

$$
\lim _{m \rightarrow \infty}\left[\psi_{m}\right]_{W^{s, \frac{n}{s}}\left(\mathbb{R}^{n}\right)} \leq \lim _{m \rightarrow \infty} C\left(\frac{1}{\log m}\right)^{1-\frac{s}{n}}=0,
$$

as claimed.

Observe that technically speaking such a sequence $\left(\psi_{m}\right)_{m \in \mathbb{N}}$ does not belong to $C_{c}^{\infty}\left(\mathbb{R}^{n}\right)$. However, this is a minor issue, that can be easily sorted by convolution. We take $\rho \in C_{0}^{\infty}\left(\mathbb{R}^{n}\right)$ a standard Friedrichs mollifier supported on $B_{1}(0)$, then by defining

$$
\varphi_{m}=\psi_{m} * \rho,
$$

we get the desired conclusion, thanks to the properties of convolutions.

The next technical result is the counterpart of Lemma 4.3 for the case $s p=n$. In particular, the space of Hölder functions now has to be replaced by a suitable Campanato space.

Lemma 5.2 Let $s \in(0,1]$ and $n \geq 1$ be such that $s<n$. Let $\left(u_{m}\right)_{m \in \mathbb{N}} \subset C_{c}^{\infty}\left(\mathbb{R}^{n}\right)$ be a Cauchy sequence with respect to $[\cdot]_{W^{s, n / s}\left(\mathbb{R}^{n}\right)}$. Then there exists another sequence $\left(\widetilde{u}_{m}\right)_{m \in \mathbb{N}} \subset$ $C_{c}^{\infty}\left(\mathbb{R}^{n}\right)$ such that:

- we have

$$
\lim _{m \rightarrow \infty}\left[u_{m}-\tilde{u}_{m}\right]_{W^{s, \frac{n}{s}}\left(\mathbb{R}^{n}\right)}=0,
$$

- $\widetilde{u}_{m}$ converges in $L_{\text {loc }}^{\frac{n}{s}}\left(\mathbb{R}^{n}\right)$ to a function $u \in \mathcal{L}^{\frac{n}{s}, n}\left(\mathbb{R}^{n}\right)$.

Moreover, this function $u$ is such that

$$
\int_{B_{1}(0)} u d x=0
$$


and we have

$$
\begin{aligned}
& \lim _{m \rightarrow \infty}\left[\tilde{u}_{m}-u\right]_{W^{s, \frac{n}{s}}\left(\mathbb{R}^{n}\right)}=0, \quad[u]_{W^{s, \frac{n}{s}}\left(\mathbb{R}^{n}\right)}=\lim _{m \rightarrow \infty}\left[u_{m}\right]_{W^{s, \frac{n}{s}}\left(\mathbb{R}^{n}\right)}, \\
& {[u]_{\mathcal{L}^{\frac{n}{s}, n}\left(\mathbb{R}^{n}\right)} \leq C[u]_{W^{s, \frac{n}{s}}\left(\mathbb{R}^{n}\right)} .}
\end{aligned}
$$

Proof The proof is similar to that of Lemma 4.3. For every $m \in \mathbb{N}$, we choose a natural number $M_{m} \geq m$ large enough, so that

$$
\left|\int_{B_{1}(0)} u_{m} d x\right|\left(\frac{1}{\log M_{m}}\right)^{1-\frac{s}{n}} \leq \frac{1}{m},
$$

and consider the sequence $\left(\varphi_{m}\right)_{m \in \mathbb{N}}$ given by Lemma 5.1. By construction we have that $M_{m}$ diverges to $+\infty$, as $m$ goes to $\infty$. Then we define

$$
\tilde{u}_{m}(x)=u_{m}(x)-\frac{1}{\left|B_{1}(0)\right|}\left(\int_{B_{1}(0)} u_{m} d x\right) \varphi_{M_{m}}(x), \quad \text { for } m \in \mathbb{N}
$$

where $\varphi_{M_{m}}$ is as in Lemma 5.1.

It is not difficult to see that

$$
\int_{B_{1}(0)} \tilde{u}_{m} d x=0
$$

We now show that $\widetilde{u}_{m}$ has the claimed properties. Thanks to the choice of $M_{m}$ and Lemma 5.1 , we have

$$
\begin{aligned}
{\left[u_{m}-\tilde{u}_{m}\right]_{W^{s, \frac{n}{s}\left(\mathbb{R}^{n}\right)}} } & =\frac{1}{\left|B_{1}(0)\right|}\left|\int_{B_{1}(0)} u_{m} d x\right|\left[\varphi_{M_{m}}\right]_{W^{s, \frac{n}{s}}\left(\mathbb{R}^{n}\right)} \\
& \leq C\left|\int_{B_{1}(0)} u_{m} d x\right|\left(\frac{1}{\log M_{m}}\right)^{1-\frac{s}{n}} \leq \frac{C}{m},
\end{aligned}
$$

which implies that

$$
\lim _{m \rightarrow \infty}\left[u_{m}-\tilde{u}_{m}\right]_{W^{s, \frac{n}{s}}\left(\mathbb{R}^{n}\right)}=0,
$$

as desired. In particular, we get that $\left(\widetilde{u}_{m}\right)_{m \in \mathbb{N}} \subset C_{c}^{\infty}\left(\mathbb{R}^{n}\right)$ is still a Cauchy sequence with respect to the seminorm $[\cdot]_{W^{s, n / s}\left(\mathbb{R}^{n}\right)}$.

In order to infer the claimed convergence, we can apply Remark 2.6 with $x_{0}=0$ and $r=1$, so to get that $\left(\widetilde{u}_{m}\right)_{m \in \mathbb{N}}$ is a Cauchy sequence in $L^{n / s}\left(B_{R}\right)$, for every $R \geq 1$. Thus we get that there exists $u \in L_{\text {loc }}^{n / s}\left(\mathbb{R}^{n}\right)$ such that

$$
\lim _{m \rightarrow \infty}\left\|\tilde{u}_{m}-u\right\|_{L^{\frac{n}{s}}\left(B_{R}\right)}=0, \quad \text { for every } R \geq 1 .
$$

In particular, the strong convergence entails that

$$
\int_{B_{1}(0)} u d x=0, \quad u \in \mathcal{L}^{\frac{n}{s}, n}\left(\mathbb{R}^{n}\right) \quad \text { and } \quad[u]_{W^{s, \frac{n}{s}}\left(\mathbb{R}^{n}\right)}<+\infty .
$$

The first property is straightforward, by recalling (5.4). The fact that $u \in \mathcal{L}^{\frac{n}{s}, n}\left(\mathbb{R}^{n}\right)$ follows from

$$
\begin{aligned}
\int_{B_{\varrho}\left(x_{0}\right)}\left|u-u_{x_{0}, \varrho}\right|^{\frac{n}{s}} d x=\lim _{m \rightarrow \infty} \int_{B_{\varrho}\left(x_{0}\right)}\left|\tilde{u}_{m}-\left(\tilde{u}_{m}\right)_{x_{0}, \varrho}\right|^{\frac{n}{s}} d x & \leq \varrho^{n} \lim _{m \rightarrow \infty}\left[\tilde{u}_{m}\right]_{\mathcal{L}^{\frac{n}{s}, n}\left(\mathbb{R}^{n}\right)}^{\frac{n}{s}} \\
& \leq C \varrho^{n} \lim _{m \rightarrow \infty}\left[\tilde{u}_{m}\right]_{W^{s, \frac{n}{s}}\left(\mathbb{R}^{n}\right)}^{\frac{n}{s}},
\end{aligned}
$$


where we used Theorem 2.4. This permits to infer that

$$
\sup _{x_{0} \in \mathbb{R}^{n}, \varrho>0} \varrho^{-n} \int_{B_{\varrho}\left(x_{0}\right)}\left|u-u_{x_{0}, \varrho}\right|^{\frac{n}{s}} d x<+\infty,
$$

as claimed. Finally, the fact that

$$
[u]_{W^{s, \frac{n}{s}}\left(\mathbb{R}^{n}\right)}<+\infty,
$$

follows from the lower semicontinuity of the Gagliardo-Slobodeckii seminorm with respect to the strong $L^{n / s}$ convergence, which in turn follows from Fatou's Lemma.

By using that $\left(\widetilde{u}_{m}\right)_{m \in \mathbb{N}} \subset C_{c}^{\infty}\left(\mathbb{R}^{n}\right)$ is still a Cauchy sequence with respect to $[\cdot]_{W^{s, p}}\left(\mathbb{R}^{n}\right)$ and arguing as in Part 2 of the proof of Theorem 3.1, we deduce (5.2). The estimate (5.3) can then be obtained by passing to the limit in the inequality of Theorem 2.4.

Theorem 5.3 Let $s \in(0,1]$ and $n \geq 1$ be such that $s<n$. We define the quotient space

$$
\dot{W}^{s, \frac{n}{s}}\left(\mathbb{R}^{n}\right):=\frac{\left\{v \in \mathcal{L}^{\frac{n}{s}, n}\left(\mathbb{R}^{n}\right):[v]_{W^{s, \frac{n}{s}}\left(\mathbb{R}^{n}\right)}<+\infty\right\}}{\sim_{C}},
$$

where $\sim_{C}$ is the same equivalence relation as in (4.4). We still endow this space with the norm

$$
\left\|\{u\}_{C}\right\|_{\dot{W}^{s, \frac{n}{s}}\left(\mathbb{R}^{n}\right)}=[u]_{W^{s, \frac{n}{s}}\left(\mathbb{R}^{n}\right)}, \quad \text { for every } u \in \mathcal{L}^{\frac{n}{s}, n}\left(\mathbb{R}^{n}\right) \text { such that }[u]_{W^{s, \frac{n}{s}}\left(\mathbb{R}^{n}\right)}<+\infty .
$$

Then $\dot{W}^{s, \frac{n}{s}}\left(\mathbb{R}^{n}\right)$ is a Banach space and there exists a linear isometric isomorphism

$$
\mathcal{J}: \mathcal{D}^{s, \frac{n}{s}}\left(\mathbb{R}^{n}\right) \rightarrow \dot{W}^{s, \frac{n}{s}}\left(\mathbb{R}^{n}\right) .
$$

In other words, the space $\mathcal{D}^{s, \frac{n}{s}}\left(\mathbb{R}^{n}\right)$ can be identified with $\dot{W}^{s, \frac{n}{s}}\left(\mathbb{R}^{n}\right)$.

Proof The proof goes along the same lines of that of Theorem 4.4. The fact that $\dot{W}^{s, n / s}\left(\mathbb{R}^{n}\right)$ is a normed vector space is straightforward.

Let us now consider the identification of our space. We take an equivalence class

$$
U=\left\{\left(u_{m}\right)_{m \in \mathbb{N}}\right\}_{s, p} \in \mathcal{D}^{s, p}\left(\mathbb{R}^{n}\right),
$$

and apply Lemma 5.2, so to get the new sequence $\left(\widetilde{u}_{m}\right)_{m \in \mathbb{N}}$. By construction, we have

$$
U=\left\{\left(u_{m}\right)_{m \in \mathbb{N}}\right\}_{s, p}=\left\{\left(\tilde{u}_{m}\right)_{m \in \mathbb{N}}\right\}_{s, p},
$$

and by Lemma 5.2, we know that $\left(\tilde{u}_{m}\right)_{m \in \mathbb{N}}$ converges to some function

$$
u \in\left\{\varphi \in \mathcal{L}^{\frac{n}{s}, n}\left(\mathbb{R}^{n}\right):[\varphi]_{W^{s, \frac{n}{s}}\left(\mathbb{R}^{n}\right)}<+\infty\right\} .
$$

Thus we may identify $U$ with the equivalence class $\{u\}_{C}$, i. e. we define $\mathcal{J}(U)=\{u\}_{C}$. As in the case $s p>n$, it is easily seen that this is a linear isometry.

On the other hand, for every equivalence class $\{v\}_{C} \in \dot{W}^{s, p}\left(\mathbb{R}^{n}\right)$, we may select any representative $v$. Then we can construct a sequence $\left(v_{m}\right)_{m \in \mathbb{N}} \subset C_{c}^{\infty}\left(\mathbb{R}^{n}\right)$ such that

$$
\lim _{m \rightarrow \infty}\left[v_{m}-v\right]_{W^{s, p}\left(\mathbb{R}^{n}\right)}=0 .
$$

It is indeed sufficient to repeat the construction of Part 1 in the proof of Theorem 3.1, with some minor modifications. We consider a sequence of cut-off functions $\eta_{j} \in C_{c}^{\infty}\left(\mathbb{R}^{n}\right)$ with $\operatorname{supp} \eta_{j} \subset B_{j^{2}}$ such that

$$
0 \leq \eta_{j} \leq 1, \quad \eta_{j} \equiv 1 \text { on } B_{j}, \quad\left|\nabla \eta_{j}\right| \leq \frac{C}{j^{2}},
$$


and observe that by Lemma B.3, we have

$$
\lim _{j \rightarrow \infty}\left[\left(v * \rho_{m}-\bar{v}_{j, m}\right) \eta_{j}-\left(v * \rho_{m}-\bar{v}_{j, m}\right)\right]_{W^{s, p}\left(\mathbb{R}^{n}\right)}=0 .
$$

Here we used the shortcut notation

$$
\bar{v}_{j, m}=\frac{1}{\left|B_{j^{2}}(0)\right|} \int_{B_{j^{2}}(0)} v * \rho_{m} d x .
$$

Thus, for every $m \geq 1$ we can choose $j_{m} \in \mathbb{N}$ such that

$$
\left[\left(v * \rho_{m}-\bar{v}_{j_{m}, m}\right) \eta_{j_{m}}-\left(v * \rho_{m}-\bar{v}_{j_{m}, m}\right)\right]_{W^{s, p}\left(\mathbb{R}^{n}\right)} \leq \frac{1}{m} .
$$

The sequence $\left(v_{m}\right)_{m \in \mathbb{N}} \subset C_{c}^{\infty}\left(\mathbb{R}^{n}\right)$ is then given by

$$
v_{m}=\left(v * \rho_{m}-\bar{v}_{j_{m}, m}\right) \eta_{j_{m}} .
$$

Indeed, by construction we have

$$
\begin{aligned}
& {\left[v_{m}-v\right]_{W^{s, \frac{n}{s}}\left(\mathbb{R}^{n}\right)}=\left[v_{m}-\left(v-\bar{v}_{j_{m}, m}\right)\right]_{W^{s, \frac{n}{s}}\left(\mathbb{R}^{n}\right)}} \\
& \leq\left[\left(v * \rho_{m}-\bar{v}_{j_{m}, m}\right) \eta_{j_{m}}-\left(v * \rho_{m}-\bar{v}_{j_{m}, m}\right)\right]_{W^{s, p}\left(\mathbb{R}^{n}\right)} \\
& +\left[\left(v * \rho_{m}-\bar{v}_{j_{m}, m}\right)-\left(v-\bar{v}_{j_{m}, m}\right)\right]_{W^{s, p}\left(\mathbb{R}^{n}\right)} \\
& \leq \frac{1}{m}+\left[\left(v * \rho_{m}-\bar{v}_{j_{m}, m}\right)-\left(v-\bar{v}_{j_{m}, m}\right)\right]_{W^{s, p}\left(\mathbb{R}^{n}\right)} \\
& =\frac{1}{m}+\left[v * \rho_{m}-v\right]_{W^{s, p}\left(\mathbb{R}^{n}\right)} .
\end{aligned}
$$

Thus, by Lemma A.1 again, we get

$$
\lim _{m \rightarrow \infty}\left[v_{m}-v\right]_{W^{s, p}\left(\mathbb{R}^{n}\right)}=0,
$$

as claimed. Accordingly, we can identify $\{v\}_{C}$ with the equivalence class $\left\{\left(v_{m}\right)_{m \in \mathbb{N}}\right\}_{s, p}$. In other words, this proves the surjectivity of $\mathcal{J}$. The proof is complete.

Remark 5.4 In the case $s p=n$, a natural replacement for the Sobolev inequality is the Moser-Trudinger inequality. However, this holds for open sets with finite measure, see e.g. [25].

\subsection{The exceptional limit case $s=n=1$}

Observe that in the previous section we have the restriction $s<n$. Thus, in order to complete the picture in the conformal case, there is still a limiting case which is missing: the case $s=1=n$. Accordingly, the summability exponent is $p=1$, as well. This one-dimensional case is special and deserves to be treated separately.

As we will see, this situation is similar to the case $s p<n$. Indeed, we have the following result.

Theorem 5.5 (The case $s=p=n=1$ ) Let us define

$$
\dot{W}^{1,1}(\mathbb{R})\left\{u \in C_{0}(\mathbb{R}): u^{\prime} \in L^{1}(\mathbb{R})\right\},
$$

where the derivative $u^{\prime}$ is intended in the sense of distributions. Then there exists a linear isometric isomorphism

$$
\mathcal{J}: \mathcal{D}^{1,1}(\mathbb{R}) \rightarrow \dot{W}^{1,1}(\mathbb{R})
$$


In other words, the space $\mathcal{D}^{1,1}(\mathbb{R})$ can be identified with $\dot{W}^{1,1}\left(\mathbb{R}^{n}\right)$.

Proof By basic Calculus, we know that for every $u \in C_{c}^{\infty}(\mathbb{R})$ and every $z<x<y$ we have

$$
|u(x)-u(y)|=\left|\int_{x}^{y} u^{\prime}(s) d s\right| \leq \int_{x}^{y}\left|u^{\prime}(s)\right| d s,
$$

and

$$
|u(x)-u(z)|=\left|\int_{z}^{x} u^{\prime}(s) d s\right| \leq \int_{z}^{x}\left|u^{\prime}(s)\right| d s
$$

By taking the limits as $y$ goes to $+\infty$ and $z$ goes to $-\infty$ in the previous inequalities, we get

$$
|u(x)| \leq \int_{x}^{+\infty}\left|u^{\prime}(s)\right| d s \text { and }|u(x)| \leq \int_{-\infty}^{x}\left|u^{\prime}(s)\right| d s .
$$

By summing these two estimates and passing to supremum in $x$, we have an analogue to Sobolev's inequality

$$
2\|u\|_{L^{\infty}(\mathbb{R})} \leq[u]_{W^{1,1}(\mathbb{R})}, \quad \text { for every } u \in C_{c}^{\infty}\left(\mathbb{R}^{n}\right) .
$$

Therefore, every Cauchy sequence $\left(u_{m}\right)_{m \in \mathbb{N}} \subset C_{c}^{\infty}(\mathbb{R})$ in the $W^{1,1}$ seminorm is a Cauchy sequence in $C_{0}(\mathbb{R})$, as well. The latter is the Banach space of continuous functions vanishing at infinity. Thus we can infer uniform convergence of $\left(u_{m}\right)_{m \in \mathbb{N}}$ to a function $u \in C_{0}(\mathbb{R})$. Moreover, by using that $L^{1}(\mathbb{R})$ is a Banach space, we can infer convergence of $\left(u_{m}^{\prime}\right)_{m \in \mathbb{N}}$ to a function $v \in L^{1}(\mathbb{R})$. It is easily seen that it must result

$$
v=u^{\prime},
$$

thus $u \in \dot{W}^{1,1}(\mathbb{R})$. This argument permits to define the isometry $\mathcal{J}$, exactly as we did in the proof of Theorem 3.1. In order to prove the surjectivity of $\mathcal{J}$, it is sufficient to show that for every $u \in \dot{W}^{1,1}(\mathbb{R})$, there exists a sequence $\left(u_{m}\right)_{m \in \mathbb{N}} \subset C_{c}^{\infty}(\mathbb{R})$ such that

$$
\lim _{m \rightarrow \infty}\left[u_{m}-u\right]_{W^{1,1}(\mathbb{R})}=0 .
$$

This is a standard fact, we leave the details to the reader.

Remark 5.6 It is not difficult to see that inequality (5.5) is sharp. It is sufficient to take a sequence $\left(u_{m}\right)_{m \in \mathbb{N}} \subset C_{c}^{\infty}(\mathbb{R})$ such that

$$
\lim _{m \rightarrow \infty}\left(\left[u_{m}-u\right]_{W^{1,1}(\mathbb{R})}+\left\|u_{m}-u\right\|_{L^{\infty}(\mathbb{R})}\right)=0,
$$

where $u$ is the function

$$
u(x)=\max \{1-|x|, 0\} .
$$

Such a sequence can be constructed by standard convolution methods.

\section{Comments and open questions}

1. Our three embedding theorems pose the question of what are the optimal constants, and whether they are achieved by extremal functions. We specifically refer to inequality (3.1) for $s p<n$, inequality (2.9) for $s p=n$, and inequality (2.11) for $s p>n$. 
Note that in the case $s=1$, the extremals in the Gagliardo-Nirenberg-Sobolev range $1<p<n$ were found by Aubin [2] and Talenti [28]. The extremals in the Morrey range $p>n$, still for $s=1$, have been recently described by Hynd and Sauffert [15]. We do not know of any similar result in the limit case $p=n$.

2. In the case of proper subsets $\Omega \subset \mathbb{R}^{n}$ the characterization result will depend on the different options. Thus, one may take completions of $C_{c}^{\infty}(\Omega)$ with respect to:

- the full norm (but localized on $\Omega$ )

$$
\|u\|_{L^{p}(\Omega)}+[u]_{W^{s, p}(\Omega)}
$$

- the full norm (but spread all over $\mathbb{R}^{n}$ ),

$$
\|u\|_{L^{p}(\Omega)}+[u]_{W^{s, p}\left(\mathbb{R}^{n}\right)} ;
$$

- the Gagliardo-Slobodeckiř seminorm (localized on $\Omega$ )

$$
[u]_{W^{s, p}(\Omega)} ;
$$

- or the Gagliardo-Slobodeckiĭ seminorm (spread all over $\mathbb{R}^{n}$ ).

In general, the resulting spaces do not coincide. See [6, Section 2] for some comments.

Acknowledgements An anonymous referee is gratefully acknowledged for the careful reading of the manuscript and for the many useful comments. We also wish to thank Alessandro Monguzzi for having kindly drawn our attention to the paper [23]. L. Brasco was financially supported by the grant FFABR Fondo Per il Finanziamento delle attività di base, of the Italian Government. The work of D. Gómez-Castro and J. L. Vázquez were funded by grant PGC2018-098440-B-I00 from the MICINN, of the Spanish Government. The research of D. Gómez-Castro was supported by the Advanced Grant Nonlocal - CPD (Nonlocal PDEs for Complex Particle Dynamics: Phase Transitions, Patterns and Synchronization) of the European Research Council Executive Agency (ERC) under the European Union's Horizon 2020 research and innovation program (grant agreement No. 883363). J. L. Vázquez is an Honorary Professor at Universidad Complutense de Madrid.

Funding Open access funding provided by Università degli Studi di Ferrara within the CRUI-CARE Agreement.

Open Access This article is licensed under a Creative Commons Attribution 4.0 International License, which permits use, sharing, adaptation, distribution and reproduction in any medium or format, as long as you give appropriate credit to the original author(s) and the source, provide a link to the Creative Commons licence, and indicate if changes were made. The images or other third party material in this article are included in the article's Creative Commons licence, unless indicated otherwise in a credit line to the material. If material is not included in the article's Creative Commons licence and your intended use is not permitted by statutory regulation or exceeds the permitted use, you will need to obtain permission directly from the copyright holder. To view a copy of this licence, visit http://creativecommons.org/licenses/by/4.0/.

\section{Appendix A. Approximation by convolution}

Let $\rho \in C_{c}^{\infty}\left(\mathbb{R}^{n}\right)$ be a standard Friedrichs mollifier supported on the ball $B_{1}(0)$, and define the sequence of smoothing kernels

$$
\rho_{m}(x)=m^{n} \rho(m x), \quad \text { for } m \in \mathbb{N} \backslash\{0\} .
$$

In [9, Theorem 2.3], the second and third authors proved, by means of interpolation techniques, that

$$
\left\|u * \rho_{m}-u\right\|_{L^{p}\left(\mathbb{R}^{n}\right)} \leq \frac{C}{m^{s}}[u]_{W^{s, p}\left(\mathbb{R}^{n}\right)}, \quad \text { for every } u \in W^{s, p}\left(\mathbb{R}^{n}\right), m \geq 1,
$$


for a constant $C=C(n)>0$. Here, we will need a stronger result (indeed, our functions need not belong to $\left.W^{s, p}\left(\mathbb{R}^{n}\right)\right)$, but without a rate of convergence. The proof is standard routine in the theory of $L^{p}$ spaces, we recall the argument for the reader's convenience.

Lemma A.1 Let $s \in[0,1]$ and $1 \leq p<+\infty$. For every $u \in L_{\text {loc }}^{1}\left(\mathbb{R}^{n}\right)$ such that

$$
[u]_{W^{s, p}\left(\mathbb{R}^{n}\right)}<+\infty,
$$

we have

$$
\lim _{m \rightarrow \infty}\left[u * \rho_{m}-u\right]_{W^{s, p}\left(\mathbb{R}^{n}\right)}=0 .
$$

Proof We focus on the case $s \in(0,1)$, the extremal cases $s=0$ and $s=1$ being simpler and well-known. By using that

$$
\int_{\mathbb{R}^{n}} \rho_{m} d x=1, \quad \text { for every } m \geq 1,
$$

we have

$$
\begin{aligned}
{[u} & \left.* \rho_{m}-u\right]_{W^{s, p}\left(\mathbb{R}^{n}\right)}^{p} \\
& =s(1-s) \iint_{\mathbb{R}^{n} \times \mathbb{R}^{n}} \frac{\left|u * \rho_{m}(x)-u(x)-\left(u * \rho_{m}(y)-u(y)\right)\right|^{p}}{|x-y|^{n+s p}} d x d y \\
& =s(1-s) \iint_{\mathbb{R}^{n} \times \mathbb{R}^{n}} \frac{\left|\int(u(x-z)-u(x)) \rho_{m}(z) d z-\int(u(y-w)-u(y)) \rho_{m}(w) d w\right|^{p}}{|x-y|^{n+s p}} d x d y \\
& =s(1-s) \iint_{\mathbb{R}^{n} \times \mathbb{R}^{n}} \frac{\left|\iint[(u(x-z)-u(x))-(u(y-w)-u(y))] \rho_{m}(z) \rho_{m}(w) d z d w\right|^{p}}{|x-y|^{n+s p}} d x d y .
\end{aligned}
$$

By using Jensen's inequality and the fact that $\rho_{m}(z) \rho_{m}(w) d z d w$ is a probability measure, we then get

$$
\begin{aligned}
& {\left[u * \rho_{m}-u\right]_{W^{s, p}\left(\mathbb{R}^{n}\right)}^{p}} \\
& \quad \leq s(1-s) \iint_{\mathbb{R}^{n} \times \mathbb{R}^{n}} \frac{\iint|(u(x-z)-u(x))-(u(y-w)-u(y))|^{p} \rho_{m}(z) \rho_{m}(w) d z d w}{|x-y|^{n+s p}} d x d y .
\end{aligned}
$$

If we now exchange the order of integration, use that $\rho_{m}$ is supported on $B_{1 / m}(0)$ and the fact that

$$
\begin{aligned}
& \lim _{|z|,|w| \rightarrow 0}\|H(\cdot-z, \cdot-w)-H\|_{L^{p}\left(\mathbb{R}^{n} \times \mathbb{R}^{n}\right)}=0, \\
& \text { where } H(x, y)=\frac{u(x)-u(y)}{|x-y|^{\frac{n}{p}+s}} \in L^{p}\left(\mathbb{R}^{n} \times \mathbb{R}^{n}\right),
\end{aligned}
$$

we easily get the desired conclusion (A.1).

\section{Appendix B. Truncation lemmas}

In what follows, we will use the shortcut notation $B_{r}$ for $B_{r}(0)$. The aim of this section is to show that there exist smooth cut-off functions $\eta_{m}$ with

$$
\eta_{m}=1 \text { in } B_{a_{m}}, \quad \eta_{m}=0 \text { in } \mathbb{R}^{n} \backslash B_{b_{m}}, \quad 0 \leq \eta_{m} \leq 1,
$$


for suitable radii $a_{m} \leq b_{m}$ diverging to $+\infty$, such that for every $[u]_{W^{s, p}\left(\mathbb{R}^{n}\right)}<+\infty$ we have

$$
\lim _{m \rightarrow \infty}\left[\eta_{m} u-u\right]_{W^{s, p}\left(\mathbb{R}^{n}\right)}=0 .
$$

We will prove this for $s p<n$ and $s p=n$. For the case $s p>n$, we will only show that $\left[\eta_{m} u-u\right]_{W^{s, p}\left(\mathbb{R}^{n}\right)}$ is uniformly bounded: this is sufficient for our scope, since we can then apply weak compactness and a convexity trick based on Mazur's Lemma.

For convenience, we will write $\varphi_{m}=1-\eta_{m}$, and prove that

$$
\left[\varphi_{m} u\right]_{W^{s, p}\left(\mathbb{R}^{n}\right)} \rightarrow 0 .
$$

The main difficulty arises from the fact that only minimal integrability assumptions are assumed on $u$. In particular, we do not require that $u \in W^{s, p}\left(\mathbb{R}^{n}\right)$.

\section{B.1. Case $s p<n$}

For $0<s \leq 1$ and $1 \leq p<+\infty$ such that $s p<n$, we recall the definition of critical Sobolev exponent

$$
p_{s}^{\star}=\frac{n p}{n-s p} .
$$

Then we have the following technical result, which is quite useful.

Lemma B.1 (Truncation lemma s $p<n$ ) Let

$$
u \in\left\{\varphi \in L^{p_{s}^{\star}}\left(\mathbb{R}^{n}\right):[\varphi]_{W^{s, p}\left(\mathbb{R}^{n}\right)}<+\infty\right\}=: \dot{W}^{s, p}\left(\mathbb{R}^{n}\right) .
$$

If $\left(\varphi_{m}\right)_{m \in \mathbb{N}} \subset C_{0}^{\infty}\left(\mathbb{R}^{n}\right)$ is a sequence of non-negative cut-off functions such that $0 \leq \varphi_{m} \leq 1$ and

$$
\varphi_{m} \equiv 0 \quad \text { on } B_{m}, \quad \varphi_{m} \equiv 1 \quad \text { on } \mathbb{R}^{n} \backslash B_{2 m} \quad \text { and } \quad\left\|\nabla \varphi_{m}\right\|_{L^{\infty}\left(\mathbb{R}^{n}\right)} \leq \frac{C}{m},
$$

then we have

$$
\lim _{m \rightarrow \infty}\left[\varphi_{m} u\right]_{W^{s, p}\left(\mathbb{R}^{n}\right)}=0 .
$$

Proof We deal with the case $s \in(0,1)$, the case $s=1$ being much simpler. We decompose the seminorm as follows

$$
\begin{aligned}
\frac{1}{s(1-s)}\left[\varphi_{m} u\right]_{W^{s, p}\left(\mathbb{R}^{n}\right)}^{p}= & \iint_{\mathbb{R}^{n} \times \mathbb{R}^{n}} \frac{\left|\varphi_{m}(x) u(x)-\varphi_{m}(y) u(y)\right|^{p}}{|x-y|^{n+s} p} d x d y \\
= & 2 \iint_{B_{m} \times\left(B_{2 m} \backslash B_{m}\right)} \frac{\left|\varphi_{m}(y) u(y)\right|^{p}}{|x-y|^{n+s p}} d x d y \\
& +2 \iint_{B_{m} \times\left(\mathbb{R}^{n} \backslash B_{2 m}\right)} \frac{|u(y)|^{p}}{|x-y|^{n+s p}} d x d y \\
& +\iint_{\left(B_{2 m} \backslash B_{m}\right) \times\left(B_{2 m} \backslash B_{m}\right)} \frac{\left|\varphi_{m}(x) u(x)-\varphi_{m}(y) u(y)\right|^{p}}{|x-y|^{n+s p}} d x d y \\
& +2 \iint_{\left(B_{2 m} \backslash B_{m}\right) \times\left(\mathbb{R}^{n} \backslash B_{2 m}\right)} \frac{|u(x)-u(y)|^{p}}{|x-y|^{n+s p}} d x d y \\
& +\iint_{\left(\mathbb{R}^{n} \backslash B_{2 m}\right) \times\left(\mathbb{R}^{n} \backslash B_{2 m}\right)} \frac{|x-y|^{n+s p}}{\mid x-u} d y \\
= & 2 \mathcal{I}_{1}+2 \mathcal{I}_{2}+\mathcal{I}_{3}+2 \mathcal{I}_{4}+\mathcal{I}_{5} .
\end{aligned}
$$


We show that each $\mathcal{I}_{i}$ converges to 0 . We treat each integral separately.

Estimate for $\mathcal{I}_{1}$. For the first integral, we first observe that

$$
\begin{aligned}
\mathcal{I}_{1} & =\iint_{B_{m} \times\left(B_{2 m} \backslash B_{m}\right)} \frac{\left|\varphi_{m}(y) u(y)\right|^{p}}{|x-y|^{n+s p}} d x d y \\
& =\iint_{B_{m} \times\left(B_{2 m} \backslash B_{m}\right)} \frac{\left|\varphi_{m}(x)-\varphi_{m}(y)\right|^{p}|u(y)|^{p}}{|x-y|^{n+s p}} d x d y \\
& \leq \frac{C}{m^{p}} \iint_{B_{m} \times\left(B_{2 m} \backslash B_{m}\right)} \frac{|u(y)|^{p}}{|x-y|^{n+s p-p}} d x d y .
\end{aligned}
$$

By noticing that

$$
B_{m} \subset B_{3 m}(y), \quad \text { for } y \in B_{2 m} \backslash B_{m},
$$

we get

$$
\int_{B_{m}} \frac{1}{|x-y|^{n+s p-p}} d x \leq \int_{B_{3 m}(y)} \frac{1}{|x-y|^{n+s p-p}} d x \leq C m^{p-s p},
$$

and thus

$$
\mathcal{I}_{1} \leq \frac{C}{m^{s p}} \int_{B_{2 m} \backslash B_{m}}|u(y)|^{p} d y .
$$

The last integral can be estimated by Hölder's inequality

$$
\frac{1}{m^{s p}} \int_{B_{2 m} \backslash B_{m}}|u(y)|^{p} d x \leq C m^{n\left(1-\frac{p}{p_{s}^{\star}}\right)-s p}\left(\int_{B_{2 m} \backslash B_{m}}|u(y)|^{p_{s}^{\star}} d x\right)^{\frac{p}{p_{s}^{\star}}} .
$$

By observing that

$$
n\left(1-\frac{p}{p_{s}^{\star}}\right)-s p=0
$$

we conclude that $\mathcal{I}_{1}$ converges to 0 as $m$ goes to $\infty$, by using that $u \in L^{p_{s}^{\star}}\left(\mathbb{R}^{n}\right)$ and the Dominated Convergence Theorem.

Estimate for $\mathcal{I}_{2}$. As for $\mathcal{I}_{2}$, by using Fubini's Theorem and Hölder's inequality, we get

$$
\mathcal{I}_{2} \leq 2 \int_{B_{m}}\left(\int_{\mathbb{R}^{n} \backslash B_{2 m}}|u(y)|^{p_{s}^{\star}} d y\right)^{\frac{n-s p}{n}}\left(\int_{\mathbb{R}^{n} \backslash B_{2 m}}|x-y|^{-(n+s p) \frac{n}{s p}} d y\right)^{\frac{s p}{n}} d x .
$$

Observe that

$$
B_{m}(x) \subset B_{2 m}, \quad \text { for every } x \in B_{m},
$$

thus we get

$$
\int_{\mathbb{R}^{n} \backslash B_{2 m}}|x-y|^{-(n+s p) \frac{n}{s p}} d y \leq \int_{\mathbb{R}^{n} \backslash B_{m}(x)}|x-y|^{-(n+s p) \frac{n}{s p}} d y=C m^{-\frac{n^{2}}{s p}} .
$$

We then obtain

$$
\mathcal{I}_{2} \leq C m^{-n}\left(\int_{\mathbb{R}^{n} \backslash B_{2 m}}|u(y)|^{p_{s}^{\star}} d y\right)^{\frac{n-s p}{n}}\left(\int_{B_{m}} d x\right)=C\left(\int_{\mathbb{R}^{n} \backslash B_{2 m}}|u(y)|^{p_{s}^{\star}} d y\right)^{\frac{n-s p}{n}}
$$

Since we have $u \in L^{p_{s}^{\star}}\left(\mathbb{R}^{n}\right)$, the last integral converges to 0 , as $m$ goes to 0 . 
Estimate for $\mathcal{I}_{3}$. For the third integral, we have

$$
\begin{aligned}
\mathcal{I}_{3} \leq & 2^{p-1} \iint_{\left(B_{2 m} \backslash B_{m}\right) \times\left(B_{2 m} \backslash B_{m}\right)} \frac{\left|\varphi_{m}(x)-\varphi_{m}(y)\right|^{p}}{|x-y|^{n+s} p}|u(x)|^{p} d x d y \\
& +2^{p-1} \iint_{\left(B_{2 m} \backslash B_{m}\right) \times\left(B_{2 m} \backslash B_{m}\right)} \frac{|u(x)-u(y)|^{p}}{|x-y|^{n+s} p}\left|\varphi_{m}(y)\right|^{p} d x d y \\
\leq & \frac{C}{m^{p}} \iint_{\left(B_{2 m} \backslash B_{m}\right) \times\left(B_{2 m} \backslash B_{m}\right)} \frac{1}{|x-y|^{n+s p-p}|u(x)|^{p} d x d y} \\
& +2^{p-1} \iint_{\left(B_{2 m} \backslash B_{m}\right) \times\left(B_{2 m} \backslash B_{m}\right)} \frac{|u(x)-u(y)|^{p}}{|x-y|^{n+s p}} d x d y .
\end{aligned}
$$

Now, the second integral converges to 0 by the Dominated Convergence Theorem. The first one can be handled as we did for $\mathcal{I}_{1}$.

Estimate for $\mathcal{I}_{4}$. We observe that

$$
\begin{aligned}
\mathcal{I}_{4}= & \iint_{\left(B_{2 m} \backslash B_{m}\right) \times\left(\mathbb{R}^{n} \backslash B_{2 m}\right)} \frac{\left|\varphi_{m}(x) u(x)-u(y)\right|^{p}}{|x-y|^{n+s p}} d x d y \\
\leq & 2^{p-1} \iint_{\left(B_{2 m} \backslash B_{m}\right) \times\left(\mathbb{R}^{n} \backslash B_{2 m}\right)} \frac{\left|\varphi_{m}(x)-\varphi_{m}(y)\right|^{p}|u(x)|^{p}}{|x-y|^{n+s p}} d x d y \\
& +2^{p-1} \iint_{\left(B_{2 m} \backslash B_{m}\right) \times\left(\mathbb{R}^{n} \backslash B_{2 m}\right)} \frac{|u(x)-u(y)|^{p}}{|x-y|^{n+s p}} d x d y,
\end{aligned}
$$

where we used that $\varphi_{m}=1$ on the complement of $B_{2 m}$. The last integral converges to 0 , while for the first one we further decompose it as follows

$$
\begin{aligned}
& \iint_{\left(B_{2 m} \backslash B_{m}\right) \times\left(\mathbb{R}^{n} \backslash B_{2 m}\right)} \frac{\left|\varphi_{m}(x)-\varphi_{m}(y)\right|^{p}|u(x)|^{p}}{|x-y|^{n+s p}} d x d y \\
&=\iint_{\left(B_{2 m} \backslash B_{m}\right) \times\left(\mathbb{R}^{n} \backslash B_{3 m}\right)} \frac{\left|\varphi_{m}(x)-\varphi_{m}(y)\right|^{p}|u(x)|^{p}}{|x-y|^{n+s p}} d x d y \\
&+\iint_{\left(B_{2 m} \backslash B_{m}\right) \times\left(B_{3 m} \backslash B_{2 m}\right)} \frac{\left|\varphi_{m}(x)-\varphi_{m}(y)\right|^{p}|u(x)|^{p}}{|x-y|^{n+s p}} d x d y \\
& \leq C \iint_{\left(B_{2 m} \backslash B_{m}\right) \times\left(\mathbb{R}^{n} \backslash B_{3 m}\right)} \frac{|u(x)|^{p}}{|x-y|^{n+s p}} d x d y \\
&+\frac{C}{m^{p}} \iint_{\left(B_{2 m} \backslash B_{m}\right) \times\left(B_{3 m} \backslash B_{2 m}\right)} \frac{|u(x)|^{p}}{|x-y|^{n+s p-p}} d x d y
\end{aligned}
$$

For the first integral, we can observe that, if $x \in B_{2 m} \backslash B_{m}$, then

$$
B_{m}(x) \subset B_{3 m},
$$


hence $\mathbb{R}^{n} \backslash B_{3 m} \subset \mathbb{R}^{n} \backslash B_{m}(x)$. This yields

$$
\begin{aligned}
\iint_{\left(B_{2 m} \backslash B_{m}\right) \times\left(\mathbb{R}^{n} \backslash B_{3 m}\right)} & \frac{|u(x)|^{p}}{|x-y|^{n+s p}} d x d y \\
& =\int_{B_{2 m} \backslash B_{m}}|u(x)|^{p}\left(\int_{\mathbb{R}^{n} \backslash B_{3 m}} \frac{1}{|x-y|^{n+s p}} d y\right) d x \\
& \leq \int_{B_{2 m} \backslash B_{m}}|u(x)|^{p}\left(\int_{\mathbb{R}^{n} \backslash B_{m}(x)} \frac{1}{|x-y|^{n+s p}} d y\right) d x \\
& \leq \frac{C}{m^{s p}} \int_{B_{2 m} \backslash B_{m}}|u(x)|^{p} d x .
\end{aligned}
$$

The last term converges to 0 , as we have shown while estimating $\mathcal{I}_{1}$. For the other remaining integral, we can use a similar estimate as for $\mathcal{I}_{1}$. We leave the details to the reader.

Estimate for $\mathcal{I}_{5}$. This is the simplest term, we just observe that

$$
\lim _{n \rightarrow \infty} \mathcal{I}_{5}=0 \text {, }
$$

by the Dominated Convergence Theorem. This concludes the proof.

\section{B.2. Case $s p>n$}

In this case, the estimate on the truncation will be slightly worse. However, this is still sufficient for our scopes.

Lemma B.2 (Truncation lemma $s p>n$ ) Let $s \in(0,1]$ and $1 \leq p<+\infty$ be such that $s p>n$. We set $\alpha=s-n / p$ and let

$$
u \in\left\{\varphi \in C^{0, \alpha}\left(\mathbb{R}^{n}\right): \varphi(0)=0,[\varphi]_{W^{s, p}\left(\mathbb{R}^{n}\right)}<+\infty\right\} .
$$

If $\left(\varphi_{m}\right)_{m \in \mathbb{N}} \subset C_{0}^{\infty}\left(\mathbb{R}^{n}\right)$ is a sequence of non-negative cut-off functions such that $0 \leq \varphi_{m} \leq 1$ and

$$
\varphi_{m} \equiv 0 \quad \text { on } B_{m}, \quad \varphi_{m} \equiv 1 \quad \text { on } \mathbb{R}^{n} \backslash B_{2 m} \quad \text { and } \quad\left\|\nabla \varphi_{m}\right\|_{L^{\infty}\left(\mathbb{R}^{n}\right)} \leq \frac{C}{m},
$$

then we have

$$
\left[\varphi_{m} u\right]_{W^{s, p}\left(\mathbb{R}^{n}\right)} \leq C,
$$

for a constant $C>0$ not depending on $m$.

Proof The proof is similar to the previous one. We deal again with the case $s \in(0,1)$. We still decompose the $(s, p)$-seminorm as before and then use the estimate

$$
|u(x)|=|u(x)-u(0)| \leq C|x|^{s-\frac{n}{p}}, \quad \text { for every } x \in \mathbb{R}^{n},
$$

in place of the hypothesis $L^{p_{s}^{\star}}$ previously used, in order to estimate $\mathcal{I}_{1}, \mathcal{I}_{3}$ and $\mathcal{I}_{4}$. We leave the details to the reader. For $\mathcal{I}_{2}$, we observe that

$$
\mathcal{I}_{2}=\iint_{B_{m} \times\left(\mathbb{R}^{n} \backslash B_{2 m}\right)} \frac{|u(y)|^{p}}{|x-y|^{n+s p}} d x d y \leq C \iint_{B_{m} \times\left(\mathbb{R}^{n} \backslash B_{2 m}\right)} \frac{|y|^{s p-n}}{|x-y|^{n+s p}} d x d y .
$$

We then use that

$$
|x-y| \geq|y|-|x| \geq|y|-\frac{|y|}{2}=\frac{|y|}{2}, \quad \text { for } x \in B_{m}, y \in \mathbb{R}^{n} \backslash B_{2 m} .
$$


Thus we obtain

$$
\mathcal{I}_{2} \leq C \iint_{B_{m} \times\left(\mathbb{R}^{n} \backslash B_{2 m}\right)}|y|^{-2 n} d x d y \leq C .
$$

This concludes the proof.

\section{B.3. Case $s p=n$}

Here we can prove a result similar to Lemma B.1. For this, we will need the PoincaréWirtinger inequality of Lemma 2.9 and the integrability information of Lemma 2.2.

Lemma B.3 (Truncation lemma $s p=n)$ Let $s \in(0,1]$ and $n \geq 1$ be such that $s<n$. Let

$$
u \in\left\{\varphi \in \mathcal{L}^{\frac{n}{s}, n}\left(\mathbb{R}^{n}\right):[\varphi]_{W^{s, \frac{n}{s}}\left(\mathbb{R}^{n}\right)}<+\infty\right\} .
$$

If $\left(\varphi_{m}\right)_{m \in \mathbb{N}} \subset C_{0}^{\infty}\left(\mathbb{R}^{n}\right)$ is a sequence of non-negative cut-off functions such that $0 \leq \varphi_{m} \leq 1$

$$
\varphi_{m} \equiv 0 \quad \text { on } B_{m}, \quad \varphi_{m} \equiv 1 \quad \text { on } \mathbb{R}^{n} \backslash B_{m^{2}} \quad \text { and } \quad\left\|\nabla \varphi_{m}\right\|_{L^{\infty}\left(\mathbb{R}^{n}\right)} \leq \frac{C}{m^{2}},
$$

then we have

$$
\lim _{m \rightarrow \infty}\left[\varphi_{m}\left(u-\bar{u}_{m^{2}}\right)\right]_{W^{s, \frac{n}{s}\left(\mathbb{R}^{n}\right)}}=0, \quad \text { where } \bar{u}_{m^{2}}=\frac{1}{\left|B_{m^{2}}\right|} \int_{B_{m^{2}}} u d x .
$$

Proof We only consider the case $s \in(0,1)$, the local case being much simpler. We decompose the seminorm as usual

$$
\begin{aligned}
& \frac{1}{s(1-s)}\left[\varphi_{m}\left(u-\bar{u}_{m^{2}}\right)\right]_{W^{s, \frac{n}{s}}\left(\mathbb{R}^{n}\right)}^{\frac{n}{s}} \\
& =\iint_{\mathbb{R}^{n} \times \mathbb{R}^{n}} \frac{\left|\varphi_{m}(x)\left(u(x)-\bar{u}_{m^{2}}\right)-\varphi_{m}(y)\left(u(y)-\bar{u}_{m^{2}}\right)\right|^{\frac{n}{s}}}{|x-y|^{2 n}} d x d y \\
& =2 \iint_{B_{m} \times\left(B_{m^{2}} \backslash B_{m}\right)} \frac{\left|\varphi_{m}(y)\left(u(y)-\bar{u}_{m^{2}}\right)\right|^{\frac{n}{s}}}{|x-y|^{2 n}} d x d y \\
& \quad+2 \iint_{B_{m} \times\left(\mathbb{R}^{n} \backslash B_{m^{2}}\right)} \frac{\mid u(y)-\bar{u}_{\left.m^{2}\right|^{\frac{n}{s}}}}{|x-y|^{2 n}} d x d y \\
& \quad+\iint_{\left(B_{m^{2}} \backslash B_{m}\right) \times\left(B_{m^{2}} \backslash B_{m}\right)} \frac{\left|\varphi_{m}(x)\left(u(x)-\bar{u}_{m^{2}}\right)-\varphi_{m}(y)\left(u(y)-\bar{u}_{m^{2}}\right)\right|^{\frac{n}{s}}}{|x-y|^{2 n}} d x d y \\
& \quad+2 \iint_{\left(B_{m^{2}} \backslash B_{m}\right) \times\left(\mathbb{R}^{n} \backslash B_{m^{2}}\right)} \frac{\left|\varphi_{m}(x)\left(u(x)-\bar{u}_{m^{2}}\right)-\left(u(y)-\bar{u}_{m^{2}}\right)\right|^{\frac{n}{s}}}{|x-y|^{2 n}} d x d y \\
& \quad+\iint_{\left(\mathbb{R}^{n} \backslash B_{m^{2}}\right) \times\left(\mathbb{R}^{n} \backslash B_{m^{2}}\right)} \frac{|u(x)-u(y)|^{\frac{n}{s}}}{|x-y|^{2 n}} d x d y \\
& =2 \mathcal{I}_{1}+2 \mathcal{I}_{2}+\mathcal{I}_{3}+2 \mathcal{I}_{4}+\mathcal{I}_{5} .
\end{aligned}
$$

We treat each integral separately.

Estimate for $\mathcal{I}_{1}$. For the term $\mathcal{I}_{1}$, we first observe that for every $y \in B_{m^{2}} \backslash B_{m}$ and every $x \in B_{m}$, we have

$$
\left|\varphi_{m}(y)\right|=\left|\varphi_{m}(y)-\varphi_{m}(x)\right| \leq \frac{C}{m^{2}}|y-x| .
$$


This entails

$$
\begin{aligned}
\mathcal{I}_{1} & =\iint_{B_{m} \times\left(B_{m^{2}} \backslash B_{m}\right)} \frac{\left|\varphi_{m}(y)\left(u(y)-\bar{u}_{m^{2}}\right)\right|^{\frac{n}{s}}}{|x-y|^{2 n}} d x d y \\
& \leq \frac{C}{\left(m^{2}\right)^{\frac{n}{s}}} \iint_{B_{m} \times\left(B_{m^{2}} \backslash B_{m}\right)} \frac{\left|u(y)-\bar{u}_{m^{2}}\right|^{\frac{n}{s}}}{|x-y|^{2 n-\frac{n}{s}}} d x d y .
\end{aligned}
$$

By noticing that

$$
B_{m} \subset B_{m^{2}+m}(y), \quad \text { for } y \in B_{m^{2}} \backslash B_{m},
$$

we get

$$
\int_{B_{m}} \frac{1}{|x-y|^{2 n-\frac{n}{s}}} d x \leq \int_{B_{m^{2}+m}(y)} \frac{1}{|x-y|^{2 n-\frac{n}{s}}} d x \leq C\left(m^{2}\right)^{\frac{n}{s}-n},
$$

and thus

$$
\mathcal{I}_{1} \leq \frac{C}{m^{2 n}} \int_{B_{m^{2}} \backslash B_{m}}\left|u(y)-\bar{u}_{m^{2}}\right|^{\frac{n}{s}} d y .
$$

If we now apply Lemma 2.9 on the right-hand side, we get

$$
\mathcal{I}_{1} \leq C \iint_{\left(B_{m^{2}} \backslash B_{m}\right) \times B_{m^{2}}} \frac{|u(x)-u(y)|^{\frac{n}{s}}}{|x-y|^{2 n}} d x d y .
$$

By using that $[u]_{W^{s, n / s}\left(\mathbb{R}^{n}\right)}<+\infty$ and the Dominated Convergence Theorem, we get that the last term above converges to 0 , as $m$ goes to $\infty$.

Estimate for $\mathcal{I}_{2}$. This term now is quite delicate, here we need a global integrability information on $u$. We observe that for every $m \geq 2, x \in B_{m}$ and $y \in \mathbb{R}^{n} \backslash B_{m^{2}}$, we have

$$
|x-y| \geq|y|-|x| \geq|y|-\sqrt{|y|} \geq \frac{|y|}{2} .
$$

Thus we get

$$
\begin{aligned}
\mathcal{I}_{2} & \leq C m^{n} \int_{\mathbb{R}^{n} \backslash B_{m^{2}}} \frac{\left|u(y)-\bar{u}_{m^{2}}\right|^{\frac{n}{s}}}{|y|^{2 n}} d y \\
& \leq C \int_{\mathbb{R}^{n} \backslash B_{m^{2}}}|y|^{\frac{n}{2}} \frac{\mid u(y)-\bar{u}_{\left.m^{2}\right|^{\frac{n}{s}}}}{|y|^{2 n}} d y=\int_{\mathbb{R}^{n} \backslash B_{m^{2}}} \frac{\mid u(y)-\bar{u}_{\left.m^{2}\right|^{\frac{n}{s}}}}{|y|^{\frac{3 n}{2}}} d y .
\end{aligned}
$$

We now observe that for $m \geq 2,|y| \geq m^{2}$ and $0<\alpha<n / 2$, we have

$$
|y|^{\frac{3 n}{2}} \geq|y|^{\frac{n}{2}} m^{2 n} \geq|y|^{\alpha} m^{2 n},
$$

and also

$$
\begin{aligned}
|y|^{\frac{3 n}{2}} & =|y|^{n}\left(\log \left(\frac{|y|}{m^{2}}\right)\right)^{\frac{n}{s}+2}|y|^{\frac{n}{2}}\left(\log \left(\frac{|y|}{m^{2}}\right)\right)^{-\frac{n}{s}-2} \\
& \geq|y|^{n}\left(\log \left(\frac{|y|}{m^{2}}\right)\right)^{\frac{n}{s}+2} \frac{1}{C_{\alpha}}|y|^{\alpha},
\end{aligned}
$$


for a suitable constant $C_{\alpha}>1$. By combining the two previous estimates, we then obtain

$$
\begin{aligned}
|y|^{\frac{3 n}{2}} & \geq \frac{|y|^{\alpha}}{2}\left(m^{2 n}+\frac{1}{C_{\alpha}}|y|^{n}\left(\log \left(\frac{|y|}{m^{2}}\right)\right)^{\frac{n}{s}+2}\right) \\
& \geq \frac{|y|^{\alpha}}{2 C_{\alpha}}\left(m^{2 n}+|y|^{n}\left(\log \left(\frac{|y|}{m^{2}}\right)\right)^{\frac{n}{s}+2}\right) .
\end{aligned}
$$

We can then estimate the integral in the right-hand side of (B.1) as follows

$$
\begin{aligned}
\int_{\mathbb{R}^{n} \backslash B_{m^{2}}} \frac{\mid u(y)-\bar{u}_{\left.m^{2}\right|^{\frac{n}{s}}}}{|y|^{\frac{3 n}{2}}} d y & \leq 2 C_{\alpha} \int_{\mathbb{R}^{n} \backslash B_{m^{2}}} \frac{\mid u(y)-\bar{u}_{\left.m^{2}\right|^{\frac{n}{s}}}}{m^{2 n}+|y|^{n}\left(\log \frac{|y|}{m^{2}}\right)^{\frac{n}{s}+2}} \frac{d y}{|y|^{\alpha}} \\
& \leq \frac{2 C_{\alpha}}{m^{2 \alpha}} \int_{\mathbb{R}^{n}} \frac{\mid u(y)-\bar{u}_{\left.m^{2}\right|^{\frac{n}{s}}}}{m^{2 n}+|y|^{n}\left|\log \frac{|y|}{m^{2}}\right|^{\frac{n}{s}+2}} d y .
\end{aligned}
$$

By using Lemma 2.2 with $p=n / s$, we get that $\mathcal{I}_{2}$ converges to 0 , as $m$ goes to $\infty$.

Estimate for $\mathcal{I}_{3}$. For the third integral, we have

$$
\begin{aligned}
& \mathcal{I}_{3} \leq 2^{\frac{n}{s}-1} \iint_{\left(B_{m^{2}} \backslash B_{m}\right) \times\left(B_{m^{2}} \backslash B_{m}\right)} \frac{\left|\varphi_{m}(x)-\varphi_{m}(y)\right|^{\frac{n}{s}}}{|x-y|^{2 n}}\left|u(x)-\bar{u}_{m^{2}}\right|^{\frac{n}{s}} d x d y \\
& +2^{\frac{n}{s}-1} \iint_{\left(B_{m^{2}} \backslash B_{m}\right) \times\left(B_{m^{2}} \backslash B_{m}\right)} \frac{|u(x)-u(y)|^{\frac{n}{s}}}{|x-y|^{2 n}}\left|\varphi_{m}(y)\right|^{\frac{n}{s}} d x d y \\
& \leq \frac{C}{\left(m^{2}\right)^{\frac{n}{s}}} \iint_{\left(B_{m^{2}} \backslash B_{m}\right) \times\left(B_{m^{2}} \backslash B_{m}\right)} \frac{1}{|x-y|^{2 n-\frac{n}{s}}}\left|u(x)-\bar{u}_{m^{2}}\right|^{\frac{n}{s}} d x d y \\
& +2^{\frac{n}{s}-1} \iint_{\left(B_{m^{2}} \backslash B_{m}\right) \times\left(B_{m^{2}} \backslash B_{m}\right)} \frac{|u(x)-u(y)|^{\frac{n}{s}}}{|x-y|^{2 n}} d x d y .
\end{aligned}
$$

Now, the second integral converges to 0 by the Dominated Convergence Theorem. The first one can be handled as we did for $\mathcal{I}_{1}$.

Estimate for $\mathcal{I}_{4}$. Here, we observe that

$$
\begin{aligned}
& \mathcal{I}_{4}=\iint_{\left(B_{m^{2}} \backslash B_{m}\right) \times\left(\mathbb{R}^{n} \backslash B_{m^{2}}\right)} \frac{\left|\varphi_{m}(x)\left(u(x)-\bar{u}_{m^{2}}\right)-\left(u(y)-\bar{u}_{m^{2}}\right)\right|^{\frac{n}{s}}}{|x-y|^{2 n}} d x d y \\
& \leq 2^{\frac{n}{s}-1} \iint_{\left(B_{m^{2}} \backslash B_{m}\right) \times\left(\mathbb{R}^{n} \backslash B_{m^{2}}\right)} \frac{\left|\varphi_{m}(x)-\varphi_{m}(y)\right|^{\frac{n}{s}}\left|u(x)-\bar{u}_{m^{2}}\right|^{\frac{n}{s}}}{|x-y|^{2 n}} d x d y \\
& +2^{\frac{n}{s}-1} \iint_{\left(B_{m^{2}} \backslash B_{m}\right) \times\left(\mathbb{R}^{n} \backslash B_{m^{2}}\right)} \frac{|u(x)-u(y)|^{\frac{n}{s}}}{|x-y|^{2 n}} d x d y,
\end{aligned}
$$


where we used that $\varphi_{m}=1$ on the complement of $B_{m^{2}}$. The last integral converges to 0 , while the first one can be decomposed as follows:

$$
\begin{aligned}
& \iint_{\left(B_{m^{2}} \backslash B_{m}\right) \times\left(\mathbb{R}^{n} \backslash B_{m^{2}}\right)} \frac{\left|\varphi_{m}(x)-\varphi_{m}(y)\right|^{\frac{n}{s}}\left|u(x)-\bar{u}_{m^{2}}\right|^{\frac{n}{s}}}{|x-y|^{2 n}} d x d y \\
& =\iint_{\left(B_{m^{2}} \backslash B_{m}\right) \times\left(\mathbb{R}^{n} \backslash B_{2 m^{2}}\right)} \frac{\left|\varphi_{m}(x)-\varphi_{m}(y)\right|^{\frac{n}{s}}\left|u(x)-\bar{u}_{m^{2}}\right|^{\frac{n}{s}}}{|x-y|^{2 n}} d x d y \\
& +\iint_{\left(B_{m^{2}} \backslash B_{m}\right) \times\left(B_{2 m^{2}} \backslash B_{m^{2}}\right)} \frac{\left|\varphi_{m}(x)-\varphi_{m}(y)\right|^{\frac{n}{s}}\left|u(x)-\bar{u}_{m^{2}}\right|^{\frac{n}{s}}}{|x-y|^{2 n}} d x d y \\
& \leq C \iint_{\left(B_{m^{2}} \backslash B_{m}\right) \times\left(\mathbb{R}^{n} \backslash B_{2 m^{2}}\right)} \frac{\left|u(x)-\bar{u}_{m^{2}}\right|^{\frac{n}{s}}}{|x-y|^{2 n}} d x d y \\
& +\frac{C}{\left(m^{2}\right)^{\frac{n}{s}}} \iint_{\left(B_{m^{2}} \backslash B_{m}\right) \times\left(B_{2 m^{2}} \backslash B_{m^{2}}\right)} \frac{\left|u(x)-\bar{u}_{m^{2}}\right|^{\frac{n}{s}}}{|x-y|^{2 n-\frac{n}{s}}} d x d y .
\end{aligned}
$$

For the first integral, we can observe that, if $x \in B_{m^{2}} \backslash B_{m}$, then

$$
B_{m^{2}}(x) \subset B_{2 m^{2}},
$$

hence $\mathbb{R}^{n} \backslash B_{2 m^{2}} \subset \mathbb{R}^{n} \backslash B_{m^{2}}(x)$. This yields

$$
\begin{aligned}
& \iint_{\left(B_{m^{2}} \backslash B_{m}\right) \times\left(\mathbb{R}^{n} \backslash B_{2 m^{2}}\right)} \frac{\left|u(x)-\bar{u}_{m^{2}}\right|^{\frac{n}{s}}}{|x-y|^{2 n}} d x d y \\
& =\int_{B_{m^{2}} \backslash B_{m}}\left|u(x)-\bar{u}_{m^{2}}\right|^{\frac{n}{s}}\left(\int_{\mathbb{R}^{n} \backslash B_{2 m^{2}}} \frac{1}{|x-y|^{2 n}} d y\right) d x \\
& \leq \int_{B_{m^{2}} \backslash B_{m}}\left|u(x)-\bar{u}_{m^{2}}\right|^{\frac{n}{s}}\left(\int_{\mathbb{R}^{n} \backslash B_{m^{2}}(x)} \frac{1}{|x-y|^{2 n}} d y\right) d x \\
& \leq \frac{C}{\left(m^{2}\right)^{n}} \int_{B_{m^{2}} \backslash B_{m}}\left|u(x)-\bar{u}_{m^{2}}\right|^{\frac{n}{s}} d x .
\end{aligned}
$$

Proceeding as for $\mathcal{I}_{1}$, the last term converges to 0 .

For the second integral we use a similar estimate as for $\mathcal{I}_{1}$. More precisely, we observe that for $x \in B_{m^{2}}$, we have $B_{2 m^{2}} \backslash B_{m^{2}} \subset B_{3 m^{2}}(x)$. Thus we can estimate

$$
\begin{aligned}
& \frac{1}{\left(m^{2}\right)^{\frac{n}{s}}} \iint_{\left(B_{m^{2}} \backslash B_{m}\right) \times\left(B_{2 m^{2}} \backslash B_{m^{2}}\right)} \frac{\left|u(x)-\bar{u}_{m^{2}}\right|^{\frac{n}{s}}}{|x-y|^{2 n-\frac{n}{s}}} d x d y \\
& =\frac{1}{\left(m^{2}\right)^{\frac{n}{s}}} \int_{B_{m^{2}} \backslash B_{m}}\left|u(x)-\bar{u}_{m^{2}}\right|^{\frac{n}{s}}\left(\int_{B_{2 m^{2} \backslash B_{m^{2}}}} \frac{1}{|x-y|^{2 n-\frac{n}{s}}} d y\right) d x \\
& \leq \frac{1}{\left(m^{2}\right)^{\frac{n}{s}}} \int_{B_{m^{2} \backslash B_{m}}}\left|u(x)-\bar{u}_{m^{2}}\right|^{\frac{n}{s}}\left(\int_{B_{3 m^{2}(x)}} \frac{1}{|x-y|^{2 n-\frac{n}{s}}} d y\right) d x \\
& =\frac{C}{\left(m^{2}\right)^{n}} \int_{B_{m^{2}} \backslash B_{m}}\left|u(x)-\bar{u}_{m^{2}}\right|^{\frac{n}{s}} d x .
\end{aligned}
$$

The latter is again the same term previously treated. 
Estimate for $\mathcal{I}_{5}$. As usual, this is the simplest term, it results

$$
\lim _{n \rightarrow \infty} \mathcal{I}_{5}=0
$$

by the Dominated Convergence Theorem. The desired conclusion now follows.

\section{References}

1. Adams, R., Fournier, J.: Sobolev Spaces (Pure and Applied Mathematics), vol. 140, 2nd edn. Elsevier, Amsterdam (2003)

2. Aubin, T.: Problèmes isopérimétriques et espaces de Sobolev. J. Differ. Geom. 11, 573-598 (1976)

3. Bergh, J., Löfström, J.: Interpolation Spaces. An introduction: Grundlehren der Mathematischen Wissenschaften, vol. 223. Springer, Berlin (1976)

4. Bourgain, J., Brezis, H., Mironescu, P.: Another look at Sobolev spaces. In: Menaldi, J.L., Rofman, E., Sulem, A. (eds.) Optimal Control and Partial Differential Equations. A Volume in Honor of Professor Alain Bensoussan's 60th Birthday, pp. 439-455. IOS Press, Amsterdam (2001)

5. Brasco, L., Mosconi, S., Squassina, M.: Optimal decay of extremals for the fractional Sobolev inequality. Calc. Var. Partial Differ. Equ. 55, Paper No. 23, 32 pp (2016)

6. Brasco, L., Salort, A.: A note on homogeneous Sobolev spaces of fractional order. Ann. Mat. Pura Appl. (4) 198, 1295-1330 (2019)

7. Campanato, S.: Proprietà di hölderianità di alcune classi di funzioni. Ann. Scuola Norm. Sup. Pisa (3) 17, 175-188 (1963)

8. Cotsiolis, A., Tavoularis, N.: Best constants for Sobolev inequalities for higher order fractional derivatives. J. Math. Anal. Appl. 295, 225-236 (2004)

9. del Teso, F., Gómez-Castro, D., Vázquez, J.L.: Estimates on translations and Taylor expansions in fractional Sobolev spaces. Nonlinear Anal. 200, 111995 (2020)

10. Deny, J., Lions, J.-L.: Les espaces du type de Beppo-Levi. Ann. Inst. Fourier 5, 305-370 (1954)

11. Fefferman, C.: Characterizations of bounded mean oscillation. Bull. Am. Math. Soc. 77, 587-588 (1971)

12. Fefferman, C., Stein, E.M.: $H^{p}$ spaces of several variables. Acta Math. 129, 137-193 (1972)

13. Franzina, G.: Non-local torsion functions and embeddings. Appl. Anal. 98, 1811-1826 (2019)

14. Giusti, E.: Direct Methods in the Calculus of Variations. World Scientific Publishing Co. Inc., River Edge (2003)

15. Hynd, R., Seuffert, F.: Extremal Functions for Morrey's Inequality (2018) (Preprint). arxiv:1810.04393

16. John, F., Nirenberg, L.: On functions of bounded mean oscillation. Commun. Pure Appl. Math. 14, 415-426 (1961)

17. Leoni, G.: A First Course in Sobolev Spaces (Graduate Studies in Mathematics), vol. 181, 2nd edn. American Mathematical Society, Providence (2017)

18. Lions, J.-L.: Théorèmes de trace et d'interpolation. I. Ann. Scuola Norm. Sup. Pisa (3) 13, 389-403 (1959)

19. Maz'ya, V.: Sobolev Spaces with Applications to Elliptic Partial Differential Equations. Second revised and augmented edition. Grundlehren der Mathematischen Wissenschaften [Fundamental Principles of Mathematical Sciences], vol. 342. Springer, Heidelberg (2011)

20. Maz'ya, V., Shaposhnikova, T.: On the Bourgain, Brezis, and Mironescu theorem concerning limiting embeddings of fractional Sobolev spaces. J. Funct. Anal. 195, 230-238 (2002)

21. Mingione, G.: The Singular Set of Solutions to Non-Differentiable Elliptic Systems. Arch. Rational Mech. Anal., 166, 287-301 (2003)

22. Mironescu, P., Russ, E.: Traces of weighted Sobolev spaces. Old and new. Nonlinear Anal. 119, 354-381 (2015)

23. Monguzzi, A., Peloso, M.M., Salvatori, M.: Fractional Laplacian, homogeneous Sobolev spaces and their realizations. Ann. Mat. Pura Appl. (4) 199, 2243-2261 (2020)

24. Moser, J.: On Harnack's theorem for elliptic differential equations. Commun. Pure Appl. Math. 16, 577$591(1961)$

25. Parini, E., Ruf, B.: On the Moser-Trudinger inequality in fractional Sobolev-Slobodeckij spaces. J. Anal. Math. 138, 281-300 (2019)

26. Peetre, J.: Espaces d'interpolation et théorème de Soboleff. Ann. Inst. Fourier (Grenoble) 16, 279-317 (1966) 
27. Stein, E.M.: Harmonic Analysis: Real-Variable Methods, Orthogonality, and Oscillatory Integrals: Princeton Mathematical Series. Monographs in Harmonic Analysis, III, vol. 43. Princeton University Press, Princeton (1993)

28. Talenti, G.: Best constant in Sobolev inequality. Ann. Mat. Pura Appl. (4) 119, 353-372 (1976)

29. Tartar, L.: An Introduction to Sobolev Spaces and Interpolation Spaces. Lecture Notes of the Unione Matematica Italiana, vol. 3. Springer, Berlin (2007)

30. Triebel, H.: Theory of Function Spaces. Monographs in Mathematics, vol. 78. Birkhäuser, Basel (1983)

31. Vázquez, J.L.: The Dirichlet Problem for the fractional $p$-Laplacian evolution equation. J. Differ. Equ. 260(7), 6038-6056 (2016)

32. Vázquez, J.L.: The evolution fractional $p$-Laplacian equation in $\mathbb{R}^{n}$. Fundamental solution and asymptotic behaviour. Nonlinear Anal. 199, 112034 (2000)

33. Warma, M.: The fractional relative capacity and the fractional Laplacian with Neumann and Robin boundary conditions on open sets. Potential Anal. 42, 499-547 (2015)

Publisher's Note Springer Nature remains neutral with regard to jurisdictional claims in published maps and institutional affiliations. 